\title{
ON MULTILINEAR SINGULAR INTEGRALS OF CALDERÓN-ZYGMUND TYPE
}

\author{
Loukas Grafakos and Rodolfo H. Torres
}

\begin{abstract}
A variety of results regarding multilinear Calderón-Zygmund singular integral operators is systematically presented. Several tools and techniques for the study of such operators are discussed. These include new multilinear endpoint weak type estimates, multilinear interpolation, appropriate discrete decompositions, a multilinear version of Schur's test, and a multilinear version of the $T 1$ Theorem suitable for the study of multilinear pseudodifferential and translation invariant operators. A maximal operator associated with multilinear singular integrals is also introduced and employed to obtain weighted norm inequalities.
\end{abstract}

\section{Introduction}

The seminal work on singular integrals by Calderón and Zygmund, as originated in [5], and the real variable methods later developed have played a crucial and influential role in modern harmonic analysis. Singular integrals nowadays appear in partial differential equations, several complex variables, operator theory, and other areas of analysis. The theory of Calderón-Zygmund singular integrals has expanded and interacted with many areas of mathematics and, as several articles in these proceedings show, it continues to be a very strong and active area of research. Moreover, the Calderón-Zygmund theory has been over the years one of the predominant themes at the El Escorial meetings. This article attempts to present many old and new results as well as some current developments in the multilinear aspects of this theory.

2000 Mathematics Subject Classification. Primary: 42B20, 42B25; Secondary: 46B70, 47G30.

Key words. Calderón-Zygmund theory, multilinear operators, pseudodifferential operators, interpolation, $T 1$ Theorem, Littlewood-Paley theory, wavelets, Schur's test. Grafakos' research partially supported by the NSF under grant DMS 9623120.

Torres' research partially supported by the NSF under grants DMS 9696267 and DMS 0070514. 
Multilinear operators arise in numerous situations involving productlike operations. Their study is also motivated by many linear and nonlinear problems in which multilinear operators naturally appear as terms of series expansions. This last point of view was pioneered and extensively pursued by Coifman and Meyer in [13], [14], [15], [16], and [47]. See also the work of Coifman, Deng, and Meyer [8], Fabes, Jerison, and Kenig [21], and Christ and Kiselev [7] where multilinear operators are used in the study of specific problems in partial differential equations. The recent proof of the boundedness of the bilinear Hilbert transform by Lacey and Thiele [43], [44], has again ignited interest in multilinear singular integrals and also in the delicate analysis of time-frequency decompositions. The latter was introduced by C. Fefferman in $[\mathbf{2 2}]$ in this context. Although we do not attempt to describe the whole history of the subject of multilinear Calderón-Zygmund singular integrals, we retake some aspects of it from the point they were left several years ago. We refocus on the theory from a more systematic viewpoint that includes later developments as well as some new progress.

Several of the results we will describe have been developed in our articles $[\mathbf{3 6}],[\mathbf{3 7}],[\mathbf{3 8}]$, and some are scattered throughout the literature. We present all these here in a unified way. We prefer to follow an expository style and only describe some of the main ideas involved. Specific details can be found in the references given in the text. The results about maximal singular integrals, however, were first presented at this meeting and they are given here including some technical details. Additionally, some new examples have been added and extensions of results not found elsewhere in the literature have been included. Overall, our approach is intended to make readily available in the multilinear setting some of the techniques that have proved to be useful in the treatment of linear singular integrals.

Littlewood-Paley decompositions are very powerful tools in the study of function spaces and are intrinsically tied to the linear CalderónZygmund theory. Such decompositions have taken over the years the simple and elegant form of discrete wavelet expansions. To some extent, wavelets simultaneously diagonalize all linear Calderón-Zygmund operators with appropriate cancellation and sufficiently smooth kernels. Wavelets have become very practical building blocks in synthesizing the behavior of such operators as described in the books by Meyer [46] or Frazier, Jawerth, and Weiss [26]. For example, pseudodifferential operators in the Hörmander's classes can be easily studied using such techniques, [57] and [35]. We shall describe a similar approach in the multilinear setting. 
The already mentioned work on the bilinear Hilbert transform clearly shows that a more refined time-frequency analysis than the one provided by wavelets is necessary to study singular multipliers, but it is still of interest to check to what extent wavelets or Littlewood-Paley theory can be pushed in the study of multilinear operators. Along these lines, we use wavelets to associate multilinear operators with discrete tensors and we find sufficient conditions on the entries of these tensors so that the associated operators are bounded on products of $L^{p}$ spaces. Theorem 1 presents these results in the form of almost diagonal estimates. The method applies to certain multilinear pseudodifferential operators. In particular, we are also able to extend a classical multilinear multiplier result of Coifman and Meyer for $L^{p}$ spaces with $p>1$ to the full range of $H^{p}$ spaces $p>0$, see Corollary 1 .

In the process of carrying through the approach above to other spaces of functions admitting Littlewood-Paley type characterizations, we were led to study positive multilinear tensors on weighted $L^{p}$ spaces. We came up with a form of Schur's test for positive multilinear integral operators, Theorem 3, which is useful for this purpose and of interest in its own.

The almost diagonal conditions we obtain for $L^{p}$ spaces are " $p$ independent" for $p>1$. This is not surprising given the close connection with Littlewood-Paley theory. However, since the multilinear operators we study behave like multiplication, the restriction to Lebesgue spaces with exponent bigger than one in the target is then a limitation of the methods used (for $p \leq 1$, Littlewood-Paley decompositions characterize the Hardy $H^{p}$ spaces not the $L^{p}$ spaces). For example, in the bilinear case one should expect to have operators mapping $L^{p} \times L^{q}$ into $L^{r}$ where, as dictated by Hölder's inequality, $1 / p+1 / q=1 / r$. Thus, for $p, q \geq 1$ one expects to obtain an endpoint result when $p=1, q=1$, and $r=1 / 2$. Again, the recent work on the bilinear Hilbert transform provided (at least to the authors) part of the inspiration to push the previously known results on multilinear Calderón-Zygmund theory below $r=1$. To achieve this, one needs to avoid Littlewood-Paley theory and go back to arguments based on the classical Calderón-Zygmund decomposition. Such an approach was used already by Coifman and Meyer $[\mathbf{1 3}]$ in obtaining weak type estimates when $r=1$ for certain operators. The full range of exponents, however, was achieved in two recent works: of Kenig and Stein in $[42]$ and of the authors in $[\mathbf{3 8}]$. In $[\mathbf{4 2}]$ the same homogeneous multilinear multipliers studied by Coifman and Meyer for $r \geq 1$ are considered while general multilinear operators with singular kernels or $x$-dependent symbols are studied in [38]. Moreover, it is shown in Theorem 4 below that multilinear operators whose kernels satisfy standard size and 
smoothness estimates and which are bounded on a single product of $L^{p}$ spaces, must also satisfy a suitable weak endpoint estimate. This result is combined with multilinear interpolation in Theorem 5 , which says that such operators are also bounded on all products of $L^{p}$ spaces with indices satisfying the natural relationship dictated by homogeneity. We also obtain an endpoint result from a product of $L^{\infty}$ spaces into $B M O$. These results justify the name multilinear Calderón-Zygmund operators that we use; things happen just as in the linear case, where the boundedness of Calderón-Zygmund operators on one $L^{q}$ for $1<q<\infty$ implies boundedness on all $L^{q}$ spaces with $q$ in the same range, as well as weak type $(1,1)$ and $L^{\infty}$ to $B M O$ endpoint estimates.

Next, we consider the problem of finding easily verifiable necessary and sufficient conditions for multilinear operators with Calderón-Zygmund type kernels to be bounded on products of $L^{p}$ spaces. A solution to this problem is provided by the multilinear version of the $T 1$ Theorem that we give in Theorem 6 . We express this result in terms of the action of an operators and its multilinear transposes on the quintessential building blocks of Fourier analysis: the characters $x \rightarrow e^{2 \pi i x \cdot \xi}$. A different formulation of a $T 1$ Theorem for multilinear forms was given before by Christ and Journé [6]. Our version is very well-suited for a couple of applications to pseudodifferential and translation invariant operators that we describe in Corollary 2 and Corollary 3.

Finally, we introduce a maximal operator associated to truncated singular integrals which we use to obtain almost everywhere convergence results and weighted norm estimates. These results are based on a multilinear version of Cotlar's inequality, Theorem 7 , and a good- $\lambda$ inequality, Theorem 8. We conclude this article with some other recent developments in the multilinear theory and a variety of open problems.

We would like to take this opportunity to thanks our colleagues in Spain for their warm hospitality during the conference. It has been, as usual, a pleasure to participate in the recent El Escorial meeting.

\section{Examples and some previous work in the subject}

Let $\mathcal{D}\left(\mathbf{R}^{n}\right)$ be the space of $C^{\infty}$ functions with compact support and let $\mathcal{S}\left(\mathbf{R}^{n}\right)$ be the space of Schwartz rapidly decreasing smooth functions. Their duals are the spaces of distributions $\mathcal{D}^{\prime}\left(\mathbf{R}^{n}\right)$ and $\mathcal{S}^{\prime}\left(\mathbf{R}^{n}\right)$. Let $T$ be a continuous and $m$-linear operator

$$
T: \mathcal{S}\left(\mathbf{R}^{n}\right) \times \cdots \times \mathcal{S}\left(\mathbf{R}^{n}\right) \rightarrow \mathcal{S}^{\prime}\left(\mathbf{R}^{n}\right) .
$$


Every such operator $T$ has an associated Schwartz kernel $K$ so that, formally, we have

(1) $T\left(f_{1}, \ldots, f_{m}\right)(x)$

$$
=\int_{\left(\mathbf{R}^{n}\right)^{m}} K\left(x, y_{1}, \ldots, y_{m}\right) f\left(y_{1}\right) \ldots f_{m}\left(y_{m}\right) d y_{1} \ldots d y_{m} .
$$

However, the above integral is not always absolutely convergent or welldefined and, in more precise distributional language, it should be interpreted as

$$
\left\langle T\left(f_{1}, \ldots, f_{m}\right), g\right\rangle=\left\langle K, g \otimes f_{1} \otimes \cdots \otimes f_{m}\right\rangle,
$$

where we use the notation $\langle\cdot, \cdot\rangle$ for the pairing of distributions and test functions and $g \otimes f_{1} \otimes \cdots \otimes f_{m}$ for the function $\left(x, y_{1}, \ldots, y_{m}\right) \rightarrow$ $g(x) f_{1}\left(y_{1}\right) \ldots f_{m}\left(y_{m}\right)$.

Using the Fourier transform in $\mathbf{R}^{n}$,

$$
\mathcal{F}_{n}(f)=\widehat{f}(\xi)=\int_{\mathbf{R}^{n}} f(x) e^{-2 \pi i x \cdot \xi} d x
$$

we can write, at least formally,

$$
\begin{aligned}
& T\left(f_{1}, \ldots, f_{m}\right)(x) \\
= & \int_{\left(\mathbf{R}^{n}\right)^{m}} \sigma\left(x, \xi_{1}, \ldots, \xi_{m}\right) \widehat{f}_{1}\left(\xi_{1}\right) \ldots \widehat{f}_{m}\left(\xi_{m}\right) e^{2 \pi i x \cdot\left(\xi_{1}+\cdots+\xi_{m}\right)} d \xi_{1} \ldots d \xi_{m} .
\end{aligned}
$$

The symbol $\sigma\left(x, \xi_{1}, \ldots, \xi_{m}\right)$ above is related to the kernel $K$ in (1) via the identity

$$
\mathcal{F}_{m n}^{-1}(\sigma(x, \cdot, \ldots, \cdot))\left(y_{1}, \ldots, y_{m}\right)=K\left(x, y_{1}, \ldots, y_{m}\right) .
$$

We will refer to $x$-independent symbols $\sigma\left(\xi_{1}, \ldots, \xi_{m}\right)$ as (Fourier) multipliers. For such symbols, the corresponding kernel takes the simple form of a function $K_{0}$ of $m$ variable such that

$$
K\left(x, y_{1}, \ldots, y_{m}\right)=K_{0}\left(x-y_{1}, \ldots, x-y_{m}\right) .
$$

Thus, multilinear Fourier multiplier operators have the form

(2) $T\left(f_{1}, \ldots, f_{m}\right)(x)$

$$
=\int_{\left(\mathbf{R}^{n}\right)^{m}} K_{0}\left(y_{1}, \ldots, y_{m}\right) f\left(x-y_{1}\right) \ldots f_{m}\left(x-y_{m}\right) d y_{1} \ldots d y_{m} .
$$

Operators as in (2) will also be called multilinear translation invariant, even though they only commute with simultaneous translations. 
An $m$-linear operator has $m$ formal transposes defined for $j=1, \ldots, m$ by

$$
\left\langle T^{* j}\left(f_{1}, \ldots, f_{m}\right), h\right\rangle=\left\langle T\left(f_{1}, \ldots, f_{j-1}, h, f_{j+1}, \ldots, f_{m}\right), f_{j}\right\rangle .
$$

The kernel $K^{* j}$ of $T^{* j}$ is related to the kernel $K$ of $T$ via

$$
\begin{aligned}
K^{* j}\left(x, y_{1}, \ldots, y_{j-1}, y_{j}, y_{j+1},\right. & \left.\ldots, y_{m}\right) \\
& =K\left(y_{j}, y_{1}, \ldots, y_{j-1}, x, y_{j+1}, \ldots, y_{m}\right) .
\end{aligned}
$$

Some operators are most naturally given by their symbols while some others are better understood using their kernels. The symbols of the transposes of multiplier operators are easy to compute; these sometimes facilitate the analysis of the operators. For example, in the bilinear case, if an operator $T$ has symbols $\sigma(\xi, \eta)$ then the symbols of its two transposes $T^{* 1}$ and $T^{* 2}$ are given by $\sigma^{* 1}(\xi, \eta)=\sigma(-(\xi+\eta), \eta)$ and $\sigma^{* 2}(\xi, \eta)=\sigma(\xi,-(\xi+\eta))$. On the other hand, for $x$-dependent symbols, the symbols of the transpose operators are not so easy to explicitly compute. For this reason, it is occasionally convenient to work instead with the kernel and the singular integral representation of the operator.

We want to describe conditions on the operators and/or their kernels or symbols that warranty boundedness on Lebesgue and other functions spaces. The most trivial example of multilinear operator is, of course, pointwise multiplication given by the symbol $\sigma \equiv 1$. If one wants to study operators whose behavior resembles multiplication, then one should consider boundedness results of the form

$$
T: L^{q_{1}} \times \cdots \times L^{q_{m}} \rightarrow L^{q},
$$

where $1 \leq q_{j} \leq \infty$ and

$$
\frac{1}{q_{1}}+\cdots+\frac{1}{q_{m}}=\frac{1}{q},
$$

as dictated by Hölder's inequality. There are numerous examples and known results in the literature. We will just review a few of them.

(i) Smooth Fourier multipliers. Coifman and Meyer studied in the 70's [13], [14], [15], operators with symbols $\sigma\left(\xi_{1}, \ldots, \xi_{m}\right) \in$ $C^{\infty}\left(\left(\mathbf{R}^{n}\right)^{m}\right)$ satisfying the estimates

$$
\left|\partial_{\xi_{1}}^{\alpha_{1}} \ldots \partial_{\xi_{m}}^{\alpha_{m}} \sigma\right| \leq C\left(1+\left|\xi_{1}\right|+\cdots+\left|\xi_{m}\right|\right)^{-\left(\left|\alpha_{1}\right|+\cdots+\left|\alpha_{m}\right|\right)}
$$

for all multi-indices $\alpha$. The boundedness properties of these operators are

$$
L^{p_{1}}\left(\mathbf{R}^{n}\right) \times \cdots \times L^{p_{m}}\left(\mathbf{R}^{n}\right) \rightarrow L^{p}\left(\mathbf{R}^{n}\right),
$$


for all $p_{j}>1$ and $\frac{1}{p_{1}}+\cdots+\frac{1}{p_{m}}=\frac{1}{p}$. The case $p<1$ was treated in [42] and [38]. It should be noted that condition (4) introduced by Coifman and Meyer can be improved by replacing the quantity $1+\left|\xi_{1}\right|$ by $\left|\xi_{1}\right|$ in (4) without affecting any of these results.

(ii) $(m+1)$-linear singular integral forms. Christ and Journé $[\mathbf{6}]$ studied multilinear forms $U$, so that the bilinear forms obtained by fixing all but two of their arguments,

$$
\begin{aligned}
U_{i j}\left(f_{1}, \ldots, f_{i-1}, f_{i+1}, \ldots, f_{j-1}, f_{j+1}, \ldots, f_{m+1}\right) & \left(f_{i}, f_{j}\right) \\
& =U\left(f_{1}, \ldots, f_{m+1}\right),
\end{aligned}
$$

are given by $U_{i j}(f, g)=\left\langle g, T_{i j} f\right\rangle$, where $T_{i j}$ is a linear operator with a Calderón-Zygmund kernel having appropriate weak cancellations. (More precise details will be given in the section about the $T 1$ Theorem below.) In this case the following continuity property holds

$$
U:\left(L^{\infty}\left(\mathbf{R}^{n}\right)\right)^{(m-1)} \times L^{2}\left(\mathbf{R}^{n}\right) \times L^{2}\left(\mathbf{R}^{n}\right) \rightarrow \mathbf{C} .
$$

(iii) Tensor products of Calderón-Zygmund operators. The known results are not restricted only to $L^{p}$ spaces. Coifman, Lions, Meyer, and Semmes [12] studied bilinear operators given by expressions of the form

$$
\sum_{j}\left(T_{j} f\right)\left(S_{j} g\right)
$$

where the $T_{j}$ and $S_{j}$ are linear Calderón-Zygmund operators. They obtained the boundedness result

$$
L_{1}^{p} \times L_{1}^{q} \rightarrow H^{r}
$$

for $\frac{1}{p}+\frac{1}{q}=\frac{1}{r}, p, q>1$ and $r>2 / 3$. Under additional cancellation conditions on the operators depending on $r$, Coifman and Grafakos [11] established

$$
H^{p} \times H^{q} \rightarrow H^{r}
$$

for all positive $r$.

(iv) Smooth multipliers in Besov spaces. There are also results available for certain spaces of smooth functions. Coifman, Dobyinsky, and Meyer [9], Dobyinsky [20], and Youssfi [60] among others, extended the results about smooth multipliers in (i) to Besov spaces. Again additional cancellation conditions are imposed on the operators. 
(v) The bilinear Hilbert transform. As mentioned in the introduction, a renewed interest in multilinear operators arose with the results on the operator

$$
(f, g) \rightarrow \frac{1}{\pi} \text { p.v. } \int_{-\infty}^{+\infty} f(x+t) g(x-t) \frac{d t}{t}
$$

which has symbol

$$
\sigma(\xi, \eta)=-i \operatorname{sgn}(\xi-\eta)
$$

Lacey and Thiele [43], [44], proved that this operator maps

$$
L^{p}(\mathbf{R}) \times L^{q}(\mathbf{R}) \rightarrow L^{r}(\mathbf{R}),
$$

for $1 / p+1 / q=1 / r, 1<p, q \leq \infty$, and $2 / 3<r<\infty$.

(vi) Singular multipliers. The type of singularity that the symbol of the bilinear Hilbert transform presents requires a delicate timefrequency analysis. Such techniques have been recently extended to other bilinear singular multiplier operators by Gilbert and Nah$\bmod [\mathbf{3 0}],[\mathbf{3 1}]$ and also to $m$-linear operators by Muscalu, Tao, and Thiele [48]. For example, in the bilinear case the symbols satisfy the estimates

$$
\left|\partial^{\alpha} \sigma(\xi, \eta)\right| \leq C\left(\operatorname{dist}((\xi, \eta), \partial P)^{-|\alpha|},\right.
$$

where $P$ is an appropriate half-plane in the $(\xi, \eta)$-plane. The boundedness properties of these operators are

$$
L^{p}(\mathbf{R}) \times L^{q}(\mathbf{R}) \rightarrow L^{r}(\mathbf{R}),
$$

where again $1 / p+1 / q=1 / r, 1<p, q \leq \infty$, and $2 / 3<r<\infty$.

The results in (i)-(iv) depend, to some extend, on Littlewood-Paley decompositions or closely related techniques. We will push such techniques forward in the next section using discrete decompositions. The results in (v) and (vi) on the other hand, escape such an analysis but they are not unrelated. In the last section of this article we will indicate some connections with the multilinear Calderón-Zygmund operators that we study.

\section{Discrete decompositions and almost diagonal estimates}

We can discretize multilinear operators using wavelets and obtain boundedness results. For simplicity in the notation we will consider only the bilinear case and we will use the following almost orthogonal wavelets. 
Fix a function $\phi$ in $\mathcal{S}\left(\mathbf{R}^{n}\right)$ whose Fourier transform is compactly supported away from the origin. Moreover, select $\phi$ as in the works of Frazier and Jawerth [24] and [25], so that the functions $\phi_{\nu k}(x)=2^{\nu n / 2} \phi\left(2^{\nu} x-k\right)$ can be used to represent each $f$ in $L^{p}, 1<p<\infty$, via

$$
f=\sum_{\nu \in Z, k \in Z^{n}}\left\langle f, \phi_{\nu k}\right\rangle \phi_{\nu k},
$$

with convergence in $L^{p}$. In this representation, the wavelet coefficients $\left\langle f, \phi_{\nu k}\right\rangle$ satisfy

$$
\begin{aligned}
c_{p}\|f\|_{L^{p}\left(\mathbf{R}^{n}\right)} & \leq\left\|\left(\sum_{\nu}\left(\sum_{k}\left|\left\langle f, \phi_{\nu k}\right\rangle\right| 2^{\nu n / 2} \chi_{Q_{\nu k}}\right)^{2}\right)^{1 / 2}\right\|_{L^{p}\left(\mathbf{R}^{n}\right)} \\
& \leq C_{p}\|f\|_{L^{p}\left(\mathbf{R}^{n}\right)},
\end{aligned}
$$

where $\chi_{Q_{\nu k}}$ is the characteristic function of the dyadic cube with lower left corner $2^{-\nu} k$ and side length $2^{-\nu}$. The collection of functions $\left\{\phi_{\nu k}\right\}$ is not an orthogonal system, but we still call it a family of (almost orthogonal) wavelets. As usual, we can think of each element $\phi_{\nu k}$ in the family as being essentially localized on $Q_{\nu k}$; that is scale $2^{-\nu}$ and position $2^{-\nu} k$. In addition to $L^{p}$, all the spaces of functions and distributions that admit Littlewood-Paley decompositions can be characterized in terms of their wavelets coefficients.

Given a bilinear operator $T$ and a pair of functions $f$ and $g$ in spaces of functions characterized by wavelets, we can write

$$
\begin{aligned}
& f=\sum_{\nu k}\left\langle f, \phi_{\nu k}\right\rangle \phi_{\nu k}, \\
& g=\sum_{\mu l}\left\langle g, \phi_{\mu l}\right\rangle \phi_{\mu l},
\end{aligned}
$$

and then

$$
\begin{aligned}
T(f, g) & =\sum_{\lambda m}\left\langle T(f, g), \phi_{\lambda m}\right\rangle \phi_{\lambda m} \\
& =\sum_{\lambda m} \sum_{\nu k} \sum_{\mu l}\left\langle T\left(\phi_{\nu k}, \phi_{\mu l}\right), \phi_{\lambda m}\right\rangle\left\langle f, \phi_{\nu k}\right\rangle\left\langle g, \phi_{\mu l}\right\rangle \phi_{\lambda m} .
\end{aligned}
$$

Thus, we can associate to $T$ the infinite array of scalars, that we call a tensor,

$$
a(\lambda m, \nu k, \mu l)=\left\langle T\left(\phi_{\nu k}, \phi_{\mu l}\right), \phi_{\lambda m}\right\rangle .
$$


Conversely, any tensor $A=\{a(\lambda m, \nu k, \mu l)\}$ as above gives rise to a bilinear operator $T$ defined by

$$
T(f, g)=\sum_{\lambda, m} \sum_{\nu, k} \sum_{\mu, l} a(\lambda m, \nu k, \mu l)\left\langle f, \phi_{\nu k}\right\rangle\left\langle g, \phi_{\mu l}\right\rangle \phi_{\lambda m}
$$

We can obtain boundedness results for $T$ by looking at its associated tensor.

If an operator behaves like multiplication and preserves the wavelets building blocks, one can expect to have

$$
T\left(\phi_{\nu k}, \phi_{\mu l}\right) \approx \phi_{\nu k} \cdot \phi_{\mu l}
$$

and

$$
a(\lambda m, \nu k, \mu l) \approx\left\langle\phi_{\nu k} \cdot \phi_{\mu l}, \phi_{\lambda m}\right\rangle \text {. }
$$

Then, because of the orthogonality properties of wavelets, the tensor associated to such an operator will be almost diagonal. That is, its entries will get smaller as the wavelets involved have substantially different scales and positions. We will quantify these observations in a precise way in terms of estimates on the entries of $a$.

Before we state the main result in this direction, it will be illustrative to consider the kind of estimates that can be obtained when one integrates the product of three wavelets at different scales and positions. We state this in the following proposition. We denote by $\operatorname{med}(\nu, \mu, \lambda)$ one of the integer numbers $\nu, \mu, \lambda$, so that $\min (\nu, \mu, \lambda) \leq \operatorname{med}(\nu, \mu, \lambda) \leq$ $\max (\nu, \mu, \lambda)$.

Proposition 1. Let $\psi_{\nu}, \psi_{\mu}, \psi_{\lambda}$ be functions satisfying

$$
\begin{aligned}
& \left|\psi_{\nu}(x)\right| \leq C_{N} \frac{2^{\nu n / 2}}{\left(1+2^{\nu}\left|x-x_{\nu}\right|\right)^{N}}, \\
& \left|\psi_{\mu}(x)\right| \leq C_{N} \frac{2^{\mu n / 2}}{\left(1+2^{\mu}\left|x-x_{\mu}\right|\right)^{N}}, \\
& \left|\psi_{\lambda}(x)\right| \leq C_{N} \frac{2^{\lambda n / 2}}{\left(1+2^{\lambda}\left|x-x_{\lambda}\right|\right)^{N}}
\end{aligned}
$$

for some $x_{\nu}, x_{\mu}, x_{\lambda}$ in $\mathbf{R}^{n}$ and for all $N>n$. 
Then,

$$
\begin{aligned}
& \left|\int_{\mathbf{R}^{n}} \psi_{\nu}(x) \psi_{\mu}(x) \psi_{\lambda}(x) d x\right| \leq \frac{C_{N} 2^{-\max (\mu, \nu, \lambda) n / 2}}{\left(\left(1+2^{\min (\nu, \mu)}\left|2^{-\nu} k-2^{-\mu} l\right|\right)\right.} \\
& \quad \times \frac{2^{\operatorname{med}(\mu, \nu, \lambda) n / 2} 2^{\min (\mu, \nu, \lambda) n / 2}}{\left.\left(1+2^{\min (\mu, \lambda)}\left|2^{-\mu} l-2^{-\lambda} m\right|\right)\left(1+2^{\min (\lambda, \nu)}\left|2^{-\lambda} m-2^{-\nu} k\right|\right)\right)^{N}} .
\end{aligned}
$$

The proof of this proposition is tedious but elementary; details can be found in [36]. It clearly shows that if the $\psi_{j}$ 's are localized far away from each other, then, as expected, the integral of their products is very small. Moreover if we impose on the functions $\psi_{j}$ of Proposition 1 the kind of smoothness and cancellation that wavelets have, then this estimate can be improved. This is done in a standard way by using cancellation to subtract appropriate Taylor polynomials of the functions. The result is that when the functions have very different associated scales the integrals are even smaller. In this way, for example, one can obtain in the estimate above an extra factor of the form $2^{-(\max (\mu, \nu, \lambda)-\min (\mu, \nu, \lambda)) \varepsilon}$. This improvement is all we need to obtain the result in our main theorem below regarding almost diagonal operators.

Theorem 1. Suppose that the entries of a tensor $\{a(\lambda m, \nu k, \mu l)\}$ associated to a bilinear operator $T$ satisfy the almost diagonal estimate

$$
\begin{aligned}
& |a(\lambda m, \nu k, \mu l)| \leq \frac{C 2^{-(\max (\mu, \nu, \lambda)-\min (\mu, \nu, \lambda)) \varepsilon}}{\left(\left(1+2^{\min (\nu, \mu)}\left|2^{-\nu} k-2^{-\mu} l\right|\right)\right.} \\
& \quad \times \frac{2^{\operatorname{med}(\mu, \nu, \lambda) n / 2} 2^{\min (\mu, \nu, \lambda) n / 2}}{\left.\left(1+2^{\min (\mu, \lambda)}\left|2^{-\mu} l-2^{-\lambda} m\right|\right)\left(1+2^{\min (\lambda, \nu)}\left|2^{-\lambda} m-2^{-\nu} k\right|\right)\right)^{N}}
\end{aligned}
$$

for some $C>0, N>n$, and $\varepsilon>0$. Then the corresponding operator $T$ can be extended to be a bounded operator from $L^{p}\left(\mathbf{R}^{n}\right) \times L^{q}\left(\mathbf{R}^{n}\right)$ into $L^{r}\left(\mathbf{R}^{n}\right)$ when $1 / p+1 / q=1 / r$ and $1<p, q, r<\infty$.

The theorem is proved following some of the ideas of Frazier and Jawerth [24] and using the vector-valued Hardy-Littlewood maximal estimates of Fefferman and Stein [23] in a crucial way. We refer again to $[\mathbf{3 6}]$ for details.

In order to verify the estimates in the theorem above on a particular operator, we want to check its action on a pair of wavelets. We introduce the notion of bilinear smooth molecules. These are functions localized at two different scales which possess certain smoothness and cancellation properties. More precisely, a collection of functions $\left\{\psi_{\mu l, \lambda m}\right\}$, with $\mu, \lambda \in$ 
$Z$ and $l, m \in Z^{n}$ is a family of bilinear smooth molecules for $L^{p}$ if

$$
\left|\partial^{\gamma} \psi_{\mu l, \lambda m}(x)\right| \leq \frac{C_{N, \gamma} 2^{\mu n / 2} 2^{\lambda n / 2} \max \left(2^{\mu}, 2^{\lambda}\right)^{|\gamma|}}{\left(1+2^{\mu}\left|x-2^{-\mu} l\right|\right)^{N}\left(1+2^{\lambda}\left|x-2^{-\lambda} m\right|\right)^{N}}
$$

and

$$
\int_{\mathbf{R}^{n}} \psi_{\mu l, \lambda m}(x) d x=0
$$

for all $\mu, \lambda, l$, and $m$. Note that estimates (6) on a bilinear molecule are clearly satisfied by the product of two wavelets $\phi_{\mu l}$ and $\phi_{\lambda m}$. It is not hard to check that operators that map pairs of wavelets into bilinear molecules are almost diagonal and, hence, bounded on product of $L^{p}$ spaces. We have from $[\mathbf{3 6}]$,

Theorem 2. Let $T$ be a bilinear operator so that $\left\{T\left(\phi_{\mu l}, \phi_{\lambda m}\right)\right\}$, $\left\{T^{* 1}\left(\phi_{\mu l}, \phi_{\lambda m}\right)\right\}$, and $\left\{T^{* 2}\left(\phi_{\mu l}, \phi_{\lambda m}\right)\right\}$ are families of bilinear smooth molecules. Then, $T: L^{p}\left(\mathbf{R}^{n}\right) \times L^{q}\left(\mathbf{R}^{n}\right) \rightarrow L^{r}\left(\mathbf{R}^{n}\right)$ for $1 / p+1 / q=1 / r$ and $1<p, q, r<\infty$.

This approach to bilinear operators applies to several classes of bilinear pseudodifferential operators of the form

$$
T(f, g)(x)=\int_{\mathbf{R}^{n}} \int_{\mathbf{R}^{n}} \sigma(x, \xi, \eta) \widehat{f}(\xi) \widehat{g}(\eta) e^{2 \pi i x \cdot(\xi+\eta)} d \xi d \eta .
$$

In fact a simple integration by parts argument shows that the functions $T\left(\phi_{\mu l}, \phi_{\lambda m}\right)$ satisfy the estimates corresponding to bilinear molecules if the symbols $\sigma$ merely satisfy the conditions

$$
\left|\partial_{x}^{\alpha} \partial_{\xi}^{\beta} \partial_{\eta}^{\gamma} \sigma(x, \xi, \eta)\right| \leq C_{\alpha, \beta, \gamma}|\xi|^{-|\beta|}|\eta|^{-|\gamma|}(|\xi|+|\eta|)^{|\alpha|},
$$

for all $\alpha, \beta, \gamma n$-tuples of nonnegative integers. The cancellation conditions on the molecules are in general not satisfied by pseudodifferential operators and they need to be imposed as we will see in the next corollary. Alternative weaker cancellations involving $B M O$ conditions will be described in Section 6. Nevertheless, we are able with our methods to treat the multipliers of Coifman and Meyer described in (i) of the previous section, and also more general operators with $x$-dependent symbols.

Moreover, the wavelets decomposition and the characterization in (5) generalize to $H^{p}$ spaces and actually to all Triebel-Lizorkin and Besov spaces. See the books by Triebel [59] and Peetre [51] for properties of these spaces and the ones by Meyer [46] and Frazier, Jawerth, and Weiss [26] for details about their wavelets characterizations. Theorem 1 and Theorem 2 can be extended to other spaces of functions. For brevity in the exposition we do not present such extensions, but we state one 
of their consequences, the extension of the results of Coiman and Meyer in (i) to $H^{p}$ spaces.

Corollary 1. Assume that $0<p, q, r<\infty, 1 / p+1 / q=1 / r$, and $r \leq 1$. Let $\sigma(\xi, \eta)$ be a $C^{\infty}$ function on $\mathbf{R}^{n} \times \mathbf{R}^{n}-\{(0,0)\}$ satisfying

$$
\left|\partial_{\xi}^{\gamma} \partial_{\eta}^{\beta} \sigma(\xi, \eta)\right| \leq C_{\gamma, \beta}(|\xi|+|\eta|)^{-|\gamma|-|\beta|},
$$

for all $n$-tuples of nonnegative integers $\gamma$ and $\beta$, and the cancellation conditions

$$
\partial_{\xi}^{\rho}(\sigma(\xi,-\xi))=\partial_{\xi}^{\rho}(\sigma(\xi, 0))=\partial_{\xi}^{\rho}(\sigma(0, \xi))=0,
$$

for all $|\rho| \leq L=\left[n\left(\frac{1}{r}-1\right)\right]+1$. Then the corresponding bilinear operator $T$ with symbol $\sigma$ is bounded from $H^{p}\left(\mathbf{R}^{n}\right) \times H^{q}\left(\mathbf{R}^{n}\right)$ into $H^{r}\left(\mathbf{R}^{n}\right)$.

We note that this class of operators is closed under the transpose operation and that the cancellation conditions on the symbols produce the required cancellation on the bilinear molecules. For $1<p, q, r<\infty$ this result is due to Coifman and Meyer and the cancellation condition is not needed. For $p=q=2$ the cancellation is necessary to map into the Besov space $\dot{B}_{1}^{0,1}\left(\mathbf{R}^{n}\right)$ as proved by Coifman, Dobyinsky and Meyer [9]; see also the work of Youssfi [60]. For other results involving $H^{p}$ spaces and singular multipliers in dimension $n=1$ see the article by Gilbert and Nahmod [29].

In the rest of this article we will focus on a class of operators which are invariant under taking transposes and have $p$-independent boundedness properties. This is consistent with the properties of the almost diagonal estimates. Such operators will be given by singular Calderón-Zygmund type kernels. Before we discuss them we present a byproduct of our work that applies to positive multilinear operators.

\section{The multilinear Schur test (a byproduct)}

Certain Besov spaces can be characterized using wavelets coefficients in such a way that the corresponding middle expression in (5) takes the form of a weighted $l^{p}$ norm on the wavelets coefficients. For example, for $f \in \dot{B}_{p}^{\alpha, p}$,

$$
f=\sum_{\nu \in Z, k \in Z^{n}}\left\langle f, \phi_{\nu k}\right\rangle \phi_{\nu k}
$$

one has that the coefficients of $f$ satisfy

$$
c\|f\|_{B_{p}^{\alpha, p}} \leq\left(\sum_{\nu} \sum_{k}\left(\left|\left\langle f, \phi_{\nu k}\right\rangle\right| 2^{\nu(\alpha+n / 2-n / p)}\right)^{p}\right)^{1 / p} \leq C\|f\|_{B_{p}^{\alpha, p} .}
$$


Since the almost diagonal estimates depend only on size, we can reduce the study of multilinear operators on Besov spaces to the study of positive tensors on $l^{p}$ spaces (even though the singular integral operators themselves are not positive). We can readily obtain boundedness results on products of Besov spaces using a multilinear Schur's test. For brevity, we only state here a version of this test for general measure spaces which is of interest in its own.

Theorem 3. Let $S$ be a positive multilinear operator. Let $X, X_{1}, \ldots, X_{m}$ be $\sigma$-finite measure spaces with non-negative measures, and let $1<$ $q, q_{1}, \ldots, q_{m}<\infty$ be numbers that satisfy (3). The following are equivalent.

(a) $S$ maps $L^{q_{1}}\left(X_{1}\right) \times \cdots \times L^{q_{m}}\left(X_{m}\right)$ to $L^{q}(X)$ with norm less than or equal to $A$.

(b) For all $B>A$ there exist measurable functions $h_{j}$ on $X_{j}$ with $0<h_{1}, \ldots, h_{m}<\infty$ a.e., such that

$S^{* j}\left(h_{1}, \ldots, h_{j-1}, S\left(h_{1}, \ldots, h_{m}\right)^{q-1}, h_{j+1}, \ldots, h_{m}\right) \leq B^{q} h_{j}^{q_{j}-1} \quad$ a.e.

for all $1 \leq j \leq n$.

(c) For all $B>A$ there exist measurable functions $u_{j}$ on $X_{j}$ and $w$ on $X$ with $0<u_{1}, \ldots, u_{m}, w<\infty$ a.e., such that

$$
\begin{aligned}
& S\left(u_{1}^{q_{1}^{\prime}}, u_{2}^{q_{2}^{\prime}}, \ldots, u_{m}^{q_{m}^{\prime}}\right) \leq B w^{q^{\prime}} \quad \text { a.e. } \\
& S^{* 1}\left(w^{q}, u_{2}^{q_{2}^{\prime}}, \ldots, u_{m}^{q_{m}^{\prime}}\right) \leq B u_{1}^{q_{1}} \quad \text { a.e. } \\
& \vdots \\
& S^{* m}\left(u_{1}^{q_{1}^{\prime}}, u_{2}^{q_{2}^{\prime}}, \ldots, w^{q}\right) \leq B u_{m}^{q_{m}} \quad \text { a.e. }
\end{aligned}
$$

The conditions in (b) are motivated by the work of Howard and Schep [40]. Another multilinear form of Schur's test was obtained by Cwikel and Kerman [17] in terms of a different and independent set of $3 m+5$ conditions involving $(m+1)(m+2)$ auxiliary functions. That (c) implies the boundedness of the operator $S$ was also recently obtained by Bekollé, Bonami, Peloso, and Ricci [1]. This follows from an application of Hölder's inequality as in the linear case. The necessity of the conditions in (c) is, however, substantially more complicated than in the linear case. Certain auxiliary functions need to be constructed and ideas of Gagliardo [28] are used. The details can be found in [37] where a brief historical account of the linear Schur test is presented. We also 
note that if in (c) we add the extra condition

$$
\int_{X} S\left(u_{1}^{q_{1}}, \ldots, u_{m}^{q_{m}}\right)(x) w^{q}(x) d x \leq B
$$

then we obtain boundedness in the off-diagonal case

$$
\frac{1}{q}>\sum_{j=1}^{m} \frac{1}{q_{j}}
$$

We give a simple application of this test to the positive $m$-linear Hilbert operator

$$
S\left(f_{1}, \ldots, f_{m}\right)(x)=\int_{0}^{\infty} \ldots \int_{0}^{\infty} \frac{f_{1}\left(x_{1}\right) \ldots f_{m}\left(x_{m}\right)}{\left(x+x_{1}+\cdots+x_{m}\right)^{m}} d x_{1} \ldots d x_{m} .
$$

Just observe that $S$ coincides with all of its transposes and that the functions

$$
u_{j}\left(x_{j}\right)=x_{j}^{-1 / q_{j} q_{j}^{\prime}}, \quad \text { and } \quad w(x)=x^{-1 / q q^{\prime}}
$$

satisfy the conditions in (c). Hence, $S$ maps $L^{q_{1}}(0, \infty) \times \cdots \times L^{q_{m}}(0, \infty)$ into $L^{q}(0, \infty)$.

Applications of the test to multilinear multipliers can be found in [37].

\section{Calderón-Zygmund kernels, interpolation, and endpoint estimates}

Let $T$ be a multilinear operator initially defined on smooth functions. Assume that the restriction of its distributional kernel away from the diagonal $x=y_{1}=y_{2}=\cdots=y_{m}$ in $\left(\mathbf{R}^{n}\right)^{m+1}$, coincides with a function, still denoted by $K$, satisfying the size estimate

$$
\left|K\left(y_{0}, y_{1}, \ldots, y_{m}\right)\right| \leq \frac{A}{\left(\sum_{k, l=0}^{m}\left|y_{k}-y_{l}\right|\right)^{m n}},
$$

the smoothness estimate, for some positive $\epsilon$,

$$
\begin{aligned}
\left|K\left(y_{0}, \ldots, y_{j}, \ldots, y_{m}\right)-K\left(y_{0}, \ldots, y_{j}^{\prime}, \ldots, y_{m}\right)\right| \\
\quad \leq \frac{A\left|y_{j}-y_{j}^{\prime}\right|^{\varepsilon}}{\left(\sum_{k, l=0}^{m}\left|y_{k}-y_{l}\right|\right)^{m n+\varepsilon}},
\end{aligned}
$$

whenever $0 \leq j \leq m$ and $\left|y_{j}-y_{j}^{\prime}\right| \leq \frac{1}{2} \max _{0 \leq k \leq m}\left|y_{j}-y_{k}\right|$, and such that

$T\left(f_{1}, \ldots, f_{m}\right)(x)=\int_{\left(\mathbf{R}^{n}\right)^{m}} K\left(x, y_{1}, \ldots, y_{m}\right) f_{1}\left(y_{1}\right) \ldots f_{m}\left(y_{m}\right) d y_{1} \ldots d y_{m}$ 
whenever $f_{1}, \ldots, f_{m} \in \mathcal{D}\left(\mathbf{R}^{n}\right)$ and $x \notin \cap_{j=1}^{m} \operatorname{supp} f_{j}$. In particular, the above conditions imply that for $f_{1}, \ldots, f_{m}, g$ in $\mathcal{D}\left(\mathbf{R}^{n}\right)$ with $\cap_{j=1}^{m} \operatorname{supp} f_{j} \cap \operatorname{supp} g=\emptyset$, we have

$$
\begin{aligned}
& \left\langle T\left(f_{1}, \ldots, f_{m}\right), g\right\rangle=\left\langle K, g \otimes f_{1} \otimes \cdots \otimes f_{m}\right\rangle \\
& =\int_{\mathbf{R}^{n}} \int_{\left(\mathbf{R}^{n}\right)^{m}} K\left(x, y_{1}, \ldots, y_{m}\right) g(x) f\left(y_{1}\right) \ldots f_{m}\left(y_{m}\right) d y_{1} \ldots d y_{m} d x,
\end{aligned}
$$

as an absolutely convergent integral.

Under the above assumptions we will says that $T$ is an $m$-linear operator with Calderón-Zygmund kernel $K$. The collection of functions satisfying (9) and (10) with parameters $m, A$, and $\varepsilon$ will be denoted by $m-C Z K(A, \varepsilon)$. Note that if $K$ is in $m-C Z K(A, \epsilon)$, then so are functions $K^{* j}$ associated with the transposes of $T$. Note also that the smoothness assumptions are clearly satisfied if, for example,

$$
\left|\partial_{y_{j}} K\left(y_{0}, \ldots, y_{m}\right)\right| \leq \frac{A}{\left(\sum_{k, l=0}^{m}\left|y_{k}-y_{l}\right|\right)^{m n+1}} .
$$

Operators with Calderón-Zygmund kernels are plentiful. Simple examples are the bilinear Riesz transforms on $\mathbf{R} \times \mathbf{R}$, given for $j=1,2$ by

$$
R_{j}\left(f_{1}, f_{2}\right)(x)=\text { p.v. } \int_{\mathbf{R}} \int_{\mathbf{R}} \frac{x-y_{j}}{\left|\left(x-y_{1}, x-y_{2}\right)\right|^{3}} f_{1}\left(y_{1}\right) f_{2}\left(y_{2}\right) d y_{1} d y_{2} .
$$

By homogeneity considerations, multilinear operators with CalderónZygmund kernels may map $T: L^{q_{1}} \times \cdots \times L^{q_{m}} \rightarrow L^{q}$, only when

$$
\frac{1}{q_{1}}+\cdots+\frac{1}{q_{m}}=\frac{1}{q}
$$

It follows form the work of Coifman and Meyer in the 70's that general operators of the form

$$
\begin{aligned}
& T_{K}\left(f_{1}, \ldots, f_{m}\right)(x) \\
& \quad=\text { p.v. } \int K\left(y_{1}, \ldots, y_{m}\right) f_{1}\left(x-y_{1}\right), \ldots, f_{m}\left(x-y_{m}\right) d y_{1}, \ldots, d y_{m}
\end{aligned}
$$

with smooth homogeneous kernels of degree $-m n$ having mean zero on the unit sphere map

$$
L^{q_{1}} \times \cdots \times L^{q_{m}} \rightarrow L^{q},
$$

for indices satisfying $1<q_{1}, \ldots, q_{m}, q<\infty$ and (11).

For linear Calderón-Zygmund singular integrals one has the classical endpoint estimate $L^{1} \rightarrow L^{1, \infty}$. Coifman and Meyer also proved a week 
estimate in the multilinear case when $q=1,[\mathbf{1 3}],[\mathbf{1 4}]$, and, as pointed out in $[\mathbf{4 2}]$, they also treated the case when $(m-1)$ of the $q_{1}$ are infinity, $[\mathbf{1 5}],[\mathbf{1 6}]$. Actually, more can be proved. For $m$-linear operators, the endpoint result is from the $m$-fold product of $L^{1}$ spaces into $L^{1 / m, \infty}$. One can also show using the above example of the bilinear Riesz transforms that, in general, the corresponding strong type $L^{1 / m}$ estimate is not possible. The most complete weak endpoint result is as follows.

Theorem 4. Let $T$ be a multilinear operator with kernel $K$ in $m-C Z K(A, \varepsilon)$. Assume that for some numbers $1 \leq q_{1}, q_{2}, \ldots, q_{m} \leq \infty$ and some $0<q<\infty$ satisfying (11), $T$ maps $L^{q_{1}} \times \cdots \times L^{q_{m}}$ into $L^{q, \infty}$. Then $T$ can be extended to a bounded operator from the $m$-fold product $L^{1} \times \cdots \times L^{1}$ into $L^{1 / m, \infty}$. Moreover, for some constant $C_{n, m}$ (that depends only on the parameters indicated) we have that

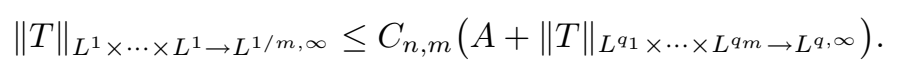

This theorem is proved using the Calderón-Zygmund decomposition, which is applied to each of the functions in the arguments of the operator at an appropriate height. Details can be found in [38]. For interpolation purposes we have kept track of the constants in the estimates of the operator norms as indicated. We mention again that for operators given by homogeneous kernels, a weak type estimate was also obtained by Kenig and Stein $[\mathbf{4 2}]$.

One single estimate provides boundedness for all possible values of the parameters given by (11) as the next theorem shows. We will use the notation $L_{c}^{\infty}$ for the space of $L^{\infty}$ functions with compact support.

Theorem 5. Let $T$ be a multilinear operator with kernel $K$ in $m-C Z K(A, \varepsilon)$. Let $1 \leq q_{1}, q_{2}, \ldots, q_{m}, q<\infty$ be given numbers satisfying (11). Suppose that either (i) or (ii) below hold:

(i) $T$ maps $L^{q_{1}, 1} \times \cdots \times L^{q_{m}, 1}$ into $L^{q, \infty}$ if $q>1$,

(ii) $T$ maps $L^{q_{1}, 1} \times \cdots \times L^{q_{m}, 1}$ into $L^{1}$ if $q=1$.

Let $p, p_{j}$ be numbers satisfying $1 / m \leq p<\infty, 1 \leq p_{j} \leq \infty$, and $\frac{1}{p}=\frac{1}{p_{1}}+\cdots+\frac{1}{p_{m}}$. Then all the statements below are valid:

(iii) when all $p_{j}>1$, then $T$ can be extended to a bounded operator from $L^{p_{1}} \times \cdots \times L^{p_{m}}$ into $L^{p}$, where $L^{p_{k}}$ should be replaced by $L_{c}^{\infty}$ if some $p_{k}=\infty$;

(iv) when some $p_{j}=1$, then $T$ can be extended to a bounded map from $L^{p_{1}} \times \cdots \times L^{p_{m}}$ into $L^{p, \infty}$, where again $L^{p_{k}}$ should be replaced by $L_{c}^{\infty}$ if some $p_{k}=\infty$;

(v) when all $p_{j}=\infty$, then $T$ can be extended to a bounded map from the $m$-fold product $L_{c}^{\infty} \times \cdots \times L_{c}^{\infty}$ into BMO. 
Moreover, there exists a constant $C_{n, m, p_{j}, q_{i}}$ such that under either assumption (i) or (ii), we have the estimate

$$
\|T\|_{L^{p_{1}} \times \cdots \times L^{p_{m}} \rightarrow L^{p}} \leq C_{n, m, p_{j}, q_{i}}(A+B),
$$

where $B=\|T\|_{L^{q_{1}} \times \cdots \times L^{q_{m}} \rightarrow L^{q, \infty}}$ if $q>1$, and $B=\|T\|_{L^{q_{1}} \times \cdots \times L^{q_{m}} \rightarrow L^{1}}$ if $q=1$.

Furthermore, conclusions (iii), (iv), and (v) as well as estimate (12) are also valid for all the transposes $T^{* j}, 1 \leq j \leq m$.

To explain the theorem, a geometric description is convenient. Identify exponents $p_{1}, \ldots, p_{m}, p$ with points $\left(1 / p_{1}, \ldots, 1 / p_{m}, 1 / p\right)$ in $\mathbf{R}^{m+1}$. We need to show that the operator $T$ is bounded for $\left(1 / p_{1}, \ldots, 1 / p_{m}, 1 / p\right)$ in the convex hull of the $m+2$ points given by $E=(1,1, \ldots, 1, m)$, $O=(0,0, \ldots, 0,0), C_{1}=(1,0, \ldots, 0,1), C_{2}=(0,1, \ldots, 0,1), \ldots$, and $C_{m}=(0,0, \ldots, 1,1)$. We consider several polyhedra with these points as vertices. The equilateral polyhedron $C_{1} C_{2} \ldots C_{m}$ is contained in an $(m-1)$-dimensional plane. Fix a point $Q$ with coordinates given by $\left(1 / q_{1}, \ldots, 1 / q_{m}, 1 / q\right)$ as in the assumptions of the theorem. Then (i) says that $Q$ is in the interior of $O C_{1} \ldots C_{m}$ while assumption (ii) says that $Q$ lies in the interior of $C_{1} \ldots C_{m}$. Conclusion (iii) means that $T$ satisfies a strong type bound in the closure of $O C_{1} C_{2} \ldots C_{m}$ minus its vertices union the interior of $E C_{1} C_{2} \ldots C_{m}$. Conclusion (iv) implies that $T$ satisfies a weak type bound on the vertices $C_{1}, \ldots, C_{m}$ and on the exterior faces of $E C_{1} \ldots C_{m}$. The main idea of the proof of the theorem is to obtain appropriate bounds in each of the faces of the polyhedron $E C_{1} \ldots C_{m} O$ by reducing matters to $(m-1)$-linear operators and then proceed by induction. Using a refinement of Theorem 4 we first obtain a weak type estimate for $T$ at the point $E$. We can then show that $T$ satisfies a strong type bound in the interior of each of the $m$ faces $\mathcal{S}_{j}=O C_{1} \ldots C_{j-1} C_{j+1} \ldots C_{m}$ of $O C_{1} \ldots C_{m}$ and weak type bounds at the vertices $C_{1}, \ldots, C_{j-1}, C_{j+1}, \ldots, C_{m}$ We obtain in this way the $m+1$ starting points needed for multilinear real interpolation. See the articles of Grafakos and Kalton [32], Janson [41], and also Strichartz [55] for a reference on multilinear interpolation. For the weak type estimates on each of the edges $E C_{j}$ we use complex interpolation. Duality arguments give the results for the transposes $T^{* j}$.

The restriction to $L_{c}^{\infty}$ is of technical nature and is needed only to compute the kernel of $(m-1)$-linear operators obtained from $T$ by "freezing one variable". Nevertheless, this restriction can be removed. In fact, at the point $O$ we obtain the result $L^{\infty} \times \cdots \times L^{\infty} \rightarrow B M O$, which is the multilinear version of the theorem of Peetre [50], Spanne [52], and Stein [53] on the $L^{\infty} \rightarrow B M O$ boundedness of singular integrals. 


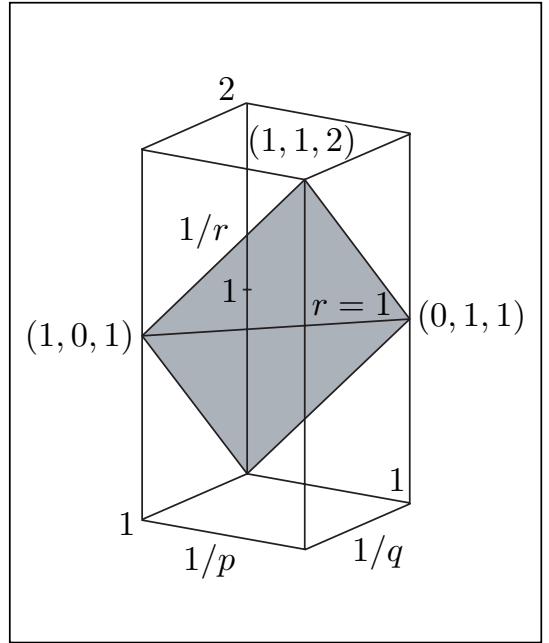

Figure 1

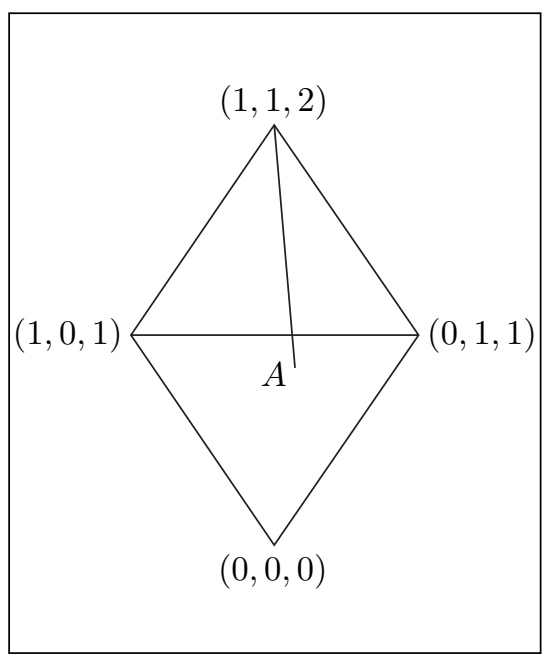

Figure 3

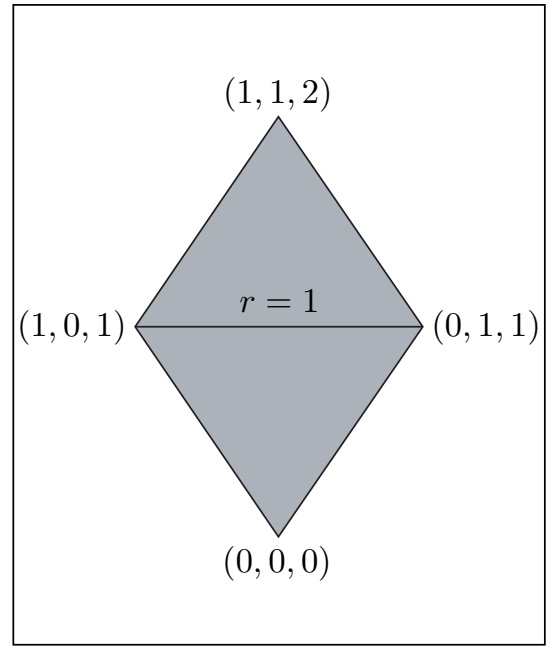

Figure 2

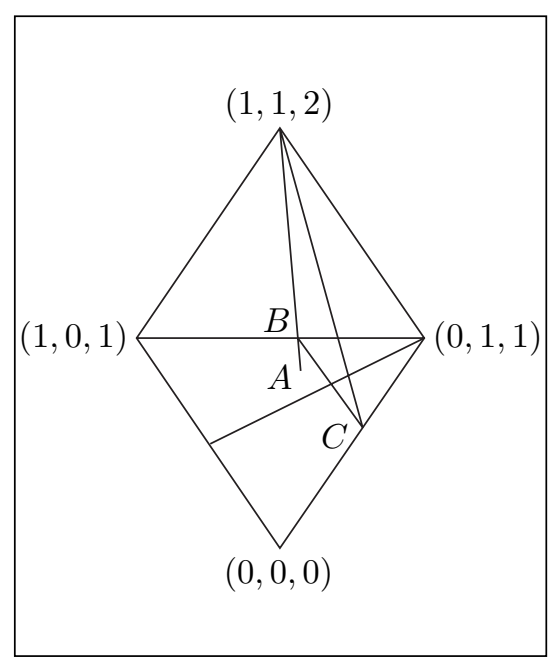

Figure 4 


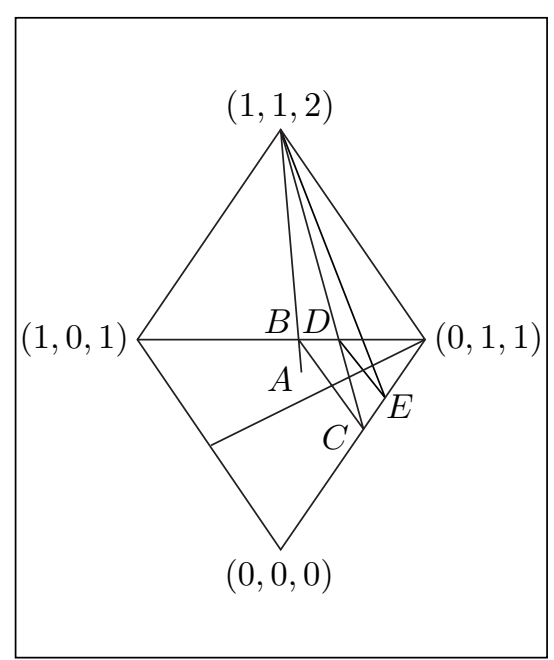

Figure 5

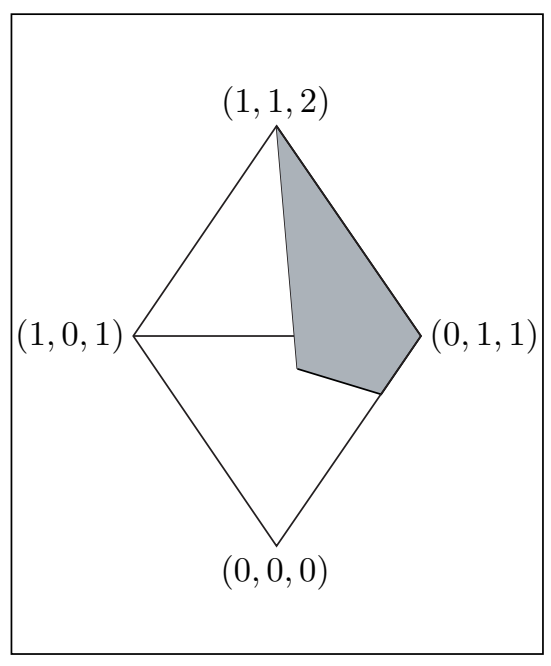

Figure 7

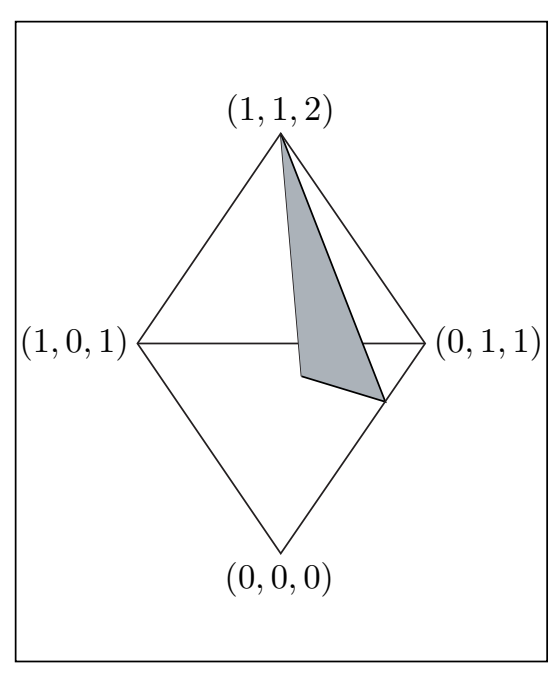

Figure 6

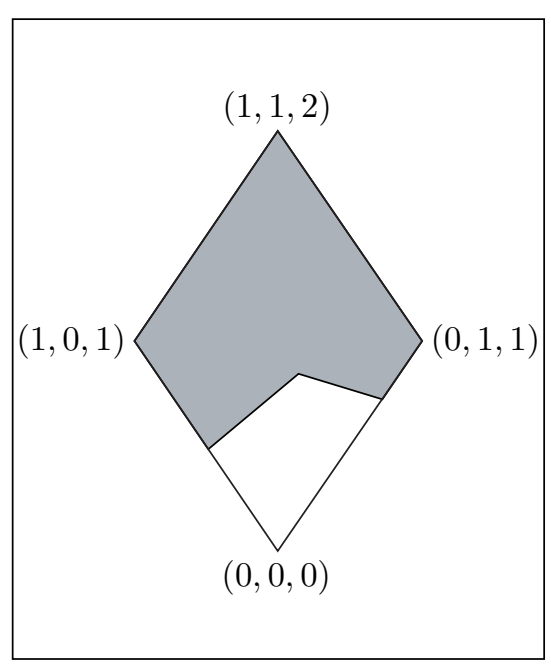

Figure 8 
Full details about the theorem are given in $[\mathbf{3 8}]$, nevertheless, we want to illustrate here some of the arguments in the bilinear case in a somehow simplified form that clearly indicates the main ideas. In this case, the region of exponents $1 / p, 1 / q$ and $1 / r$ on which we want to prove boundedness estimates is shown in Figure 1 and Figure 2, while the steps in the interpolation process are illustrated in Figures 3-8.

We explain the argument very briefly since the figures speak for themselves. We start from a strong point estimate at the point $A$ in the lower triangle in Figure 3 and by complex interpolation we obtain weak boundedness on the segment connecting $A$ with the point $(1,1,2)$ (weak boundedness at this last endpoint follows from Theorem 4). By dualizing the point $B$ we obtain restricted boundedness at the point $C$ for the transpose $T^{* 1}$. Again by complex interpolation we obtain restricted weak boundedness on the segment connecting $C$ and $(1,1,2)$ for $T^{* 1}$, Figure 4 . Using duality we get restricted weak boundedness for the point $E$ for $T$ in Figure 5. We now have three points for $T$ at which we know restricted weak boundedness. Using real multilinear interpolation we obtain strong boundedness in the region shown in Figure 6. Repeating this procedure we can get the entire region represented in Figure 7 and using a similar argument with the other transpose we get the region in Figure 8. Since the whole process works for any of the transposes of $T$, we can obtain a similar region for one of them and, again by duality, we can complete the whole picture of Figure 2. Note also that by freezing one variable we can get a point on, say, the inside of the segment from $(0,0,0)$ to $(0,1,1)$ and the linear theory gives then strong estimates on the interior of that side and a weak estimate at the endpoint $(0,1,1)$. With this point and $(1,1,2)$ we can use again complex interpolation to obtain weak estimates on the side with those two vertices. The other sides are obtained in a similar way. Finally at the point $(0,0,0)$ we get $L^{\infty} \times L^{\infty} \rightarrow B M O$.

\section{The multilinear $T 1$ theorem}

Theorem 5 say that if a multilinear operator with kernel in $m-C Z K(A, \varepsilon)$ maps $L^{q_{1}} \times \cdots \times L^{q_{m}}$ into $L^{q}$ for a single point $\left(1 / q_{1}, \ldots, 1 / q_{m}, 1 / q\right)$ with $q>1$, then it maps $L^{p_{1}} \times \cdots \times L^{p_{m}}$ into $L^{p}$ in the full range of possible exponents. A necessary and sufficient condition for this to happen is given by the following result. For convenience in the notation, we set $T^{* 0}=T$. 
Theorem 6. Fix $1<q_{1}, \ldots, q_{m}, q<\infty$ satisfying (11). Let $T$ be a continuous multilinear operator from $\mathcal{S}\left(\mathbf{R}^{n}\right) \times \cdots \times \mathcal{S}\left(\mathbf{R}^{n}\right)$ into $\mathcal{S}^{\prime}\left(\mathbf{R}^{n}\right)$ with kernel $K$ in $m-C Z K(A, \varepsilon)$. Then $T$ has a bounded extension from $L^{q_{1}} \times \cdots \times L^{q_{m}}$ into $L^{q}$ if and only if

$$
\sup _{\xi_{1} \in \mathbf{R}^{n}} \ldots \sup _{\xi_{m} \in \mathbf{R}^{n}}\left\|T^{* j}\left(e^{2 \pi i \xi_{1} \cdot(\cdot)}, \ldots, e^{2 \pi i \xi_{m} \cdot(\cdot)}\right)\right\|_{B M O} \leq B
$$

for all $j=0,1, \ldots, m$. Moreover, if (13) holds then we have that

$$
\|T\|_{L^{q_{1}} \times \cdots \times L^{q_{m}} \rightarrow L^{q}} \leq c_{n, m, q_{j}}(A+B),
$$

for some constant $c_{n, m, q_{j}}$ depending only on the parameters indicated.

The conditions in (13) are motivated by one of the well-known versions of the original $T 1$ Theorem of David and Journé [18]. The necessity of the conditions follows from Theorem 5. To show that they are sufficient, we first use the conditions and the action of $T$ and its transposes on characters (properly defined via a limiting argument) to obtain uniform BMO estimates on normalized bump functions as denoted by Stein in $[\mathbf{5 4}]$. In fact, one can obtain estimates of the form

$$
\left\|T^{* j}\left(\phi_{1}^{R_{1}, x_{1}}, \ldots, \phi_{m}^{R_{m}, x_{m}}\right)\right\|_{B M O} \leq C
$$

where the normalize bumps $\phi^{R_{j}, x_{j}}$ are functions given by $\phi^{R_{j}, x_{j}}=$ $\phi\left(\frac{x-x_{j}}{R_{j}}\right)$, and they satisfy $\operatorname{supp} \phi \subset B(0,1)$, and $\left\|\partial^{\alpha} \phi\right\|_{\infty} \leq 1$ for all $|\alpha| \leq[n / 2]+1$. The result then follows by an induction argument combined again with Theorem 5. For example, in the linear case the conditions in (14) give the estimates on $T$ and its transpose $T^{*}$,

$$
\left\|T\left(\phi^{R, z}\right)\right\|_{L^{2}\left(\mathbf{R}^{n}\right)}+\left\|T^{*}\left(\phi^{R, z}\right)\right\|_{L^{2}\left(\mathbf{R}^{n}\right)} \leq C R^{n / 2},
$$

uniformly for all normalized bumps, which is known to be equivalent to the $L^{2}$ boundedness of $T$. We remark that actually it would be enough in some cases to prove the multilinear analogue of (15) for normalized characteristic function of cubes as done in the linear case (see e.g. the work of Nazarov, Treil, and Volberg [49]), but in our general situation $T$ is only a priori defined on smooth functions.

Using different arguments, Christ and Journé established in [6] another version of the multilinear $T 1$ Theorem. Consider the $(m+1)$-linear form defined on functions in $\mathcal{D}\left(\mathbf{R}^{n}\right)$ via

$$
U\left(f_{1}, \ldots, f_{m}, f_{m+1}\right)=\left\langle T\left(f_{1}, \ldots, f_{m}\right), f_{m+1}\right\rangle .
$$


The results in [6] imply, in particular, that the estimates

$$
\left|U\left(f_{1}, \ldots, f_{m+1}\right)\right| \leq C\left(\prod_{j \neq k, l}\left\|f_{j}\right\|_{L^{\infty}}\right)\left\|f_{k}\right\|_{L^{2}}\left\|f_{l}\right\|_{L^{2}}
$$

are equivalent to certain multilinear weak-boundedness condition on $U$ (that we will not describe here), together with the hypotheses that $U_{j}(1) \in B M O$. The distributions $U_{j}(1)$ are defined by $\left\langle U_{j}(1), g\right\rangle=$ $U(1, \ldots, 1, g, 1, \ldots, 1)$, with $g$ a test function with mean zero in the $j$-position. Our version of the multilinear $T 1$ Theorem expressed in terms of the conditions (13) on characters is well-suited for several applications including the following.

We use the notation $\vec{z}=\left(z_{1}, \ldots, z_{m}\right)$ and $d \vec{z}=d z_{1} \ldots d z_{m}$. Consider multilinear pseudodifferential operators

(16) $T\left(f_{1}, \ldots, f_{m}\right)(x)$

$$
=\int_{\mathbf{R}^{n}} \cdots \int_{\mathbf{R}^{n}} \sigma(x, \vec{\xi}) \widehat{f}_{1}\left(\xi_{1}\right) \ldots \widehat{f_{m}}\left(\xi_{m}\right) e^{2 \pi i x \cdot\left(\xi_{1}+\cdots+\xi_{m}\right)} d \vec{\xi}
$$

and translation invariant operators

(17) $T\left(f_{1}, \ldots, f_{m}\right)(x)$

$$
=\int_{\mathbf{R}^{n}} \cdots \int_{\mathbf{R}^{n}} K_{0}\left(x-y_{1}, \ldots, x-y_{m}\right) f_{1}\left(y_{1}\right) \ldots f_{m}\left(y_{m}\right) d \vec{y},
$$

where the integrals should be interpreted in an appropriate manner.

Corollary 2. Let $T$ be initially defined on Schwartz functions by (16) with a symbol $\sigma$ in the class $m-S_{1,1}^{0}$, i.e.

$$
\begin{aligned}
\left|\partial_{x}^{\alpha} \partial_{\xi_{1}}^{\beta_{1}} \ldots \partial_{\xi_{m}}^{\beta_{m}} \sigma\left(x, \xi_{1}, \ldots, \xi_{m}\right)\right| & \\
& \leq C_{\alpha, \beta}\left(1+\left|\xi_{1}\right|+\cdots+\left|\xi_{m}\right|\right)^{|\alpha|-\left(\left|\beta_{1}\right|+\cdots+\left|\beta_{m}\right|\right)}
\end{aligned}
$$

for all $\alpha, \beta_{1}, \ldots, \beta_{m} n$-tuples of nonnegative integers. Suppose that all of the transposes $T^{* j}$ are also given in the form of (16) with symbols in $m-S_{1,1}^{0}$. Then $T$ extends as bounded operator from $L^{q_{1}} \times \cdots \times L^{q_{m}}$ into $L^{q}$, when $1<q_{1}, \ldots, q_{m}, q<\infty$ satisfy (11). Moreover, if we let some of the $q_{j}=1$, then $T$ maps $L^{q_{1}} \times \cdots \times L^{q_{m}}$ into $L^{q, \infty}$.

In what follows $\left|\left(u_{1}, \ldots, u_{m}\right)\right|$ will denote the Euclidean norm of $\vec{u}=$ $\left(u_{1}, \ldots, u_{m}\right)$ as an element in $\mathbf{R}^{n m}$. 
Corollary 3. Let $K_{0}\left(u_{1}, \ldots, u_{m}\right)$ be a locally integrable function on $\left(\mathbf{R}^{n}\right)^{m}-\{0\}$ which satisfies the size estimate

$$
\left|K_{0}\left(u_{1}, \ldots, u_{m}\right)\right| \leq A\left|\left(u_{1}, \ldots, u_{m}\right)\right|^{-n m},
$$

the cancellation condition

$$
\left|\int_{R_{1}<\left|\left(u_{1}, \ldots, u_{m}\right)\right|<R_{2}} K_{0}\left(u_{1}, \ldots, u_{m}\right) d \vec{u}\right| \leq A<\infty,
$$

for all $0<R_{1}<R_{2}<\infty$, and the smoothness condition

$$
\begin{aligned}
\left|K_{0}\left(u_{1}, \ldots, u_{j}, \ldots, u_{m}\right)-K_{0}\left(u_{1}, \ldots, u_{j}^{\prime}, \ldots, u_{m}\right)\right| & \\
& \leq A \frac{\left|u_{j}-u_{j}^{\prime}\right|^{\varepsilon}}{\left|\left(u_{1}, \ldots, u_{m}\right)\right|^{n m+\varepsilon}},
\end{aligned}
$$

whenever $\left|u_{j}-u_{j}^{\prime}\right|<\frac{1}{2}\left|u_{j}\right|$. Suppose that for some sequence $\varepsilon_{j} \downarrow 0$ the limit

$$
\lim _{j \rightarrow \infty} \int_{\varepsilon_{j}<|\vec{u}| \leq 1} K_{0}\left(u_{1}, \ldots, u_{m}\right) d \vec{u}
$$

exists. Let $T$ be the operator given by (17) as a principal value using the sequence $\varepsilon_{j}$ and kernel $K_{0}$. Then T maps $L^{q_{1}} \times \cdots \times L^{q_{m}}$ into $L^{q}$, when $1<q_{j}<\infty$ and (11) is satisfied. Moreover, if some of the $q_{j}=1$, then $T$ maps $L^{q_{1}} \times \cdots \times L^{q_{m}}$ into $L^{q, \infty}$.

Both corollaries follow from Theorem 6 because the operators considered have kernels in $m-C Z K$ and their actions on the characters produce uniformly bounded functions. For example, in Corollary 2, the kernel of the operators is given by

$$
K\left(x, y_{1}, \ldots, y_{m}\right)=\mathcal{F}^{-1}(a(x, \cdot, \ldots, \cdot))\left(y_{1}, \ldots, y_{m}\right)
$$

which satisfies

$$
\left|\partial^{\alpha} K\left(y_{0}, y_{1}, \ldots, y_{m}\right)\right| \leq \frac{C_{\alpha}}{\left(\sum_{k, l=0}^{m}\left|y_{k}-y_{l}\right|\right)^{m n+|\alpha|}} .
$$

In addition,

$$
T\left(e^{2 \pi i \xi_{1} \cdot(\cdot)}, \ldots, e^{2 \pi i \xi_{m} \cdot(\cdot)}\right)(x)=a\left(x, \xi_{1}, \ldots, \xi_{m}\right) e^{2 \pi i x \cdot\left(\xi_{1}+\cdots+\xi_{m}\right)},
$$

and similarly with $T^{* j}$. Corollary 2 can be interpreted as a multilinear formulation of a results of Bourdaud [4] about linear pseudodifferential operators in the so-called exotic class. In Corollary 3 the conditions (13) follow from a simple calculation. This corollary extends results of Benedek, Calderón, and Panzone [2] in the multilinear setting. Corollary 3 applies, in particular, to kernels given by homogeneous Lipschitz 
functions with mean value zero on the unit sphere $\mathbf{S}^{n m-1}$, considered by Coifman and Meyer [13] when $m=2$ and $n=1$.

\section{Maximal operator and weighted norm inequalities}

The operators with Calderón-Zygmund kernels studied in the previous section which are bounded on a product of $L^{p}$ spaces will be called multilinear Calderón-Zygmund operators. As in the linear case it is of interest to study weighted norm inequalities for such operators. To do so we introduce the truncated maximal singular integral operator

$$
T_{*}\left(f_{1}, \ldots, f_{m}\right)(x)=\sup _{\delta>0}\left|T_{\delta}\left(f_{1}, \ldots, f_{m}\right)(x)\right|,
$$

where

$$
\begin{aligned}
& T_{\delta}\left(f_{1}, \ldots, f_{m}\right)(x) \\
& \quad=\int_{\left|x-y_{1}\right|^{2}+\cdots+\left|x-y_{m}\right|^{2}>\delta^{2}} K\left(x, y_{1}, \ldots, y_{m}\right) f_{1}\left(y_{1}\right) \ldots f_{m}\left(y_{m}\right) d \vec{y} .
\end{aligned}
$$

The boundedness of $T_{*}$ will be a consequence of the following pointwise estimate which is a multilinear version of Cotlar's inequality. We will use the notation $\vec{f}=\left(f_{1}, \ldots, f_{m}\right)$.

Theorem 7. Let $T$ be an m-linear Calderón-Zygmund operator. Then for all $\vec{f}$ in any product of $L^{q_{j}}\left(\mathbf{R}^{n}\right)$ spaces, with $1 \leq q_{j}<\infty$, we have

$$
T_{*}(\vec{f})(x) \leq C\left(\left(M\left(|T(\vec{f})|^{1 / m}\right)(x)\right)^{m}+\prod_{j=1}^{m} M f_{j}(x)\right) .
$$

Actually, a better estimate also holds. Namely, for all $0<\eta$,

$$
T_{*}(\vec{f})(x) \leq C_{\eta}\left(\left(M\left(|T(\vec{f})|^{\eta}\right)(x)\right)^{1 / \eta}+\prod_{j=1}^{m} M f_{j}(x)\right) .
$$

The proof of this improved estimate will appear in [39]. For brevity, here we present a different argument which applies only for $\eta=1 / m$, but which is rather simple and still illustrates some of the ideas.

First, observe that it suffices to prove (18) for

$$
\widetilde{T}_{*}(\vec{f})(x)=\sup _{\delta>0}\left|\widetilde{T}_{\delta}\left(f_{1}, \ldots, f_{m}\right)(x)\right|,
$$


where

$$
\begin{aligned}
\widetilde{T}_{\delta}\left(f_{1}, \ldots, f_{m}\right)(x) & =\int_{\vec{y} \notin S_{\delta}(x)} K\left(x, y_{1}, \ldots, y_{m}\right) f_{1}\left(y_{1}\right) \ldots f_{m}\left(y_{m}\right) d \vec{y} \\
S_{\delta}(x) & =\left\{\vec{y}: \sup _{1 \leq j \leq m}\left|x-y_{j}\right| \leq \delta\right\}
\end{aligned}
$$

and

$$
U_{\delta}=\left\{\vec{y} \in S_{\delta}(x):\left|x-y_{1}\right|^{2}+\cdots+\left|x-y_{m}\right|^{2}>\delta^{2}\right\} .
$$

(The difference between the two operators is clearly bounded by $C \prod_{j=1}^{m} M f_{j}(x)$.)

Fix $\delta>0$ and let $B=B(x, \delta / 2)$ be the ball of center $x$ and radius $\delta / 2$. Then, for $z \in B$ we have

$$
\widetilde{T}_{\delta}(\vec{f})(z)=T(\vec{f})(z)-T\left(\overrightarrow{f_{0}}\right)(z),
$$

where $\overrightarrow{f_{0}}=\left(f_{1} \chi_{B(0, \delta)}, \ldots, f_{m} \chi_{B(0, \delta)}\right)$. Using the regularity of the kernel we obtain

(21) $\left|\widetilde{T}_{\delta}(\vec{f})(x)-\widetilde{T}_{\delta}(\vec{f})(z)\right| \leq \int_{\vec{y} \notin S_{\delta}(x)} \frac{A|x-z|^{\varepsilon} \prod_{j=1}^{m}\left|f_{j}\left(y_{j}\right)\right|}{\left(\left|x-y_{1}\right|+\cdots+\left|x-y_{m}\right|\right)^{n m+\varepsilon}} d \vec{y}$.

The region of integration in right hand side of (21) can be written as a sum of sets $R_{j_{1}, \ldots, j_{l}}$ in $\left(\mathbf{R}^{n}\right)^{m}$ so that for $\vec{y}=\left(y_{1}, \ldots, y_{m}\right) \in R_{j_{1}, \ldots, j_{l}}$ exactly the $l$ components $y_{j_{k}}$ with $j_{k} \in\left\{j_{1}, \ldots, j_{l}\right\}$ satisfy $\left|x-y_{j_{k}}\right| \leq \delta$, and we can easily estimate

$$
\begin{aligned}
& \int_{\vec{y} \in R_{j_{1}, \ldots, j_{l}}} \frac{A|x-z|^{\varepsilon}}{\left(\left|x-y_{1}\right|+\cdots+\left|x-y_{m}\right|\right)^{n m+\varepsilon}} \prod_{j=1}^{m}\left|f_{j}\left(y_{j}\right)\right| d \vec{y} \\
& \leq A \delta^{\varepsilon} \prod_{j \in\left\{j_{1}, \ldots, j_{l}\right\}} \int_{\left|x-y_{j}\right| \leq \delta}\left|f_{j}\left(y_{j}\right)\right| d y_{j} \prod_{j \notin\left\{j_{1}, \ldots, j_{l}\right\}} \int_{\left|x-y_{j}\right|>\delta} \frac{\left|f_{j}\left(y_{j}\right)\right|}{\left|x-y_{j}\right|^{\frac{n m+\varepsilon}{m-l}}} d y_{j} \\
& \leq C A \prod_{j \in\left\{j_{1}, \ldots, j_{l}\right\}} M f_{j}(x) \prod_{j \notin\left\{j_{1}, \ldots, j_{l}\right\}} \delta^{\frac{n+\varepsilon}{m-l}} \int_{\left|x-y_{j}\right|>\delta} \frac{\left|f_{j}\left(y_{j}\right)\right|}{\left|x-y_{j}\right|^{\frac{n m+\varepsilon}{m-l}}} d y_{j} \\
& \leq C A \prod_{j=1}^{m} M f_{j}(x) .
\end{aligned}
$$

Therefore, for $z$ in $B(x, \delta / 2)$

$$
\left|\widetilde{T}_{\delta}(\vec{f})(x)\right| \leq C A \prod_{j=1}^{m} M f_{j}(x)+\left|T(\vec{f})(z)-T\left(\overrightarrow{f_{0}}\right)(z)\right| .
$$


We may assume then that $\widetilde{T}_{\delta} \vec{f}(x) \neq 0$ and that there exists $\lambda$ such that

$$
0<2\left(C A \prod_{j=1}^{m} M f_{j}(x)\right)^{1 / m} \leq \lambda<\left(\widetilde{T}_{\delta} \vec{f}(x)\right)^{1 / m} ;
$$

or there is nothing to prove. Note that the above assumption implies that for $z \in B,\left|T \vec{f}(z)-T \vec{f}_{0}(z)\right|^{1 / m}>\lambda / 2$. Since $T$ is a Calderón-Zygmund operator, it satisfies also a weak estimate

$$
L^{1} \times \cdots \times L^{1} \rightarrow L^{1 / m, \infty}
$$

with a certain bound $W$. We have

$$
\begin{aligned}
&\left|\left\{z \in B(x, \delta / 2):\left|T \vec{f}_{0}(z)\right|^{1 / m}>\lambda / 4\right\}\right| \\
& \leq C W^{1 / m} \lambda^{-1}\left(\prod_{j=1}^{m}\left\|f_{j} \chi_{B(x, \delta / 2)}\right\|_{L^{1}}\right)^{1 / m} \\
& \leq C W^{1 / m} \lambda^{-1} \delta^{n}\left(\prod_{j=1}^{m} M f_{j}(x)\right)^{1 / m} .
\end{aligned}
$$

In addition, Chebychev's inequality gives

(25) $\left|\left\{z \in B(x, \delta / 2):|T \vec{f}(z)|^{1 / m}>\lambda / 4\right\}\right| \leq C \lambda^{-1} \delta^{n} M\left(|T \vec{f}|^{1 / m}\right)(x)$.

For all $\lambda$ satisfying (23), we now have

$$
\begin{aligned}
& B\left(x, \frac{\delta}{2}\right)=\left\{z \in B\left(x, \frac{\delta}{2}\right):\left|T \vec{f}_{0}(z)\right|^{1 / m}>\lambda / 4\right\} \\
& \cup\left\{z \in B\left(x, \frac{\delta}{2}\right):|T \vec{f}(z)|^{1 / m}>\lambda / 4\right\},
\end{aligned}
$$

and therefore (24) and (25) give

$$
\lambda \leq C\left(\left(\prod_{j=1}^{m} M f_{j}(x)\right)^{1 / m}+M\left(|T \vec{f}|^{1 / m}\right)(x)\right) .
$$

Taking the supremum over all $\lambda<\left(\widetilde{T}_{\delta} \vec{f}(x)\right)^{1 / m}$ in (23) we obtain estimate (18) for $\widetilde{T}_{*}$. As we have previously observed, this suffices to obtain the estimate for $T_{*}$.

An immediate corollary is the following. 
Corollary 4. Let $T$ be an m-linear Calderón-Zygmund operator. Then for indices $1<q_{1}, \ldots, q_{m} \leq \infty, q<\infty$ satisfying $1 / q_{1}+\cdots+1 / q_{m}=1 / q$ we have

$$
T_{*}: L^{q_{1}} \times \cdots \times L^{q_{m}} \rightarrow L^{q} .
$$

Also

$$
T_{*}: L^{q_{1}} \times \cdots \times L^{q_{m}} \rightarrow L^{q, \infty}
$$

when at least one $q_{j}$ is equal to one.

The improved estimate (19) is needed to obtain the result for $q<1 / m$.

The boundedness of $T_{*}$ can be used, as in the linear case, to show that if $T$ is given by

$$
T\left(f_{1}, \ldots, f_{m}\right)(x)=\lim _{\delta \rightarrow 0} \int_{\vec{y} \notin S_{\delta}(x)} K\left(x, y_{1}, \ldots, y_{m}\right) f_{1}\left(y_{1}\right) \ldots f_{m}\left(y_{m}\right) d \vec{y}
$$

for functions in $\mathcal{S}$, then the integral is actually pointwise a.e. convergent for functions $f_{j} \in L^{q_{j}}$.

In order to obtain weighted norm estimates for multilinear CalderónZygmund operators, we prove a good- $\lambda$ inequality for the associated $T_{*}$. Our approach is motivated by the work of Coifman and Fefferman [10] in the linear case.

Recall that a weight $w$ is in the class $A_{\infty}$ if there exist $c, \theta>0$ such that for every cube $Q$ and every measurable set $E \subset Q$,

$$
\frac{w(E)}{w(Q)} \leq c\left(\frac{|E|}{|Q|}\right)^{\theta}
$$

where, for a measurable set $F, w(F)=\int_{F} w(x) d x$.

Theorem 8. Let $T$ be an m-linear Calderón-Zygmund operator. Let $\vec{f}$ be in any product of $L^{q_{j}}\left(\mathbf{R}^{n}\right)$, with $1 \leq q_{j}<\infty, w \in A_{\infty}$, and let $\theta$ be as in (26). Then, for $\alpha>0$ and all $\gamma>0$ sufficiently small

$w\left(\left\{T_{*}(\vec{f})>2^{m+1} \alpha\right\} \bigcap\left\{\prod_{j=1}^{m} M f_{j} \leq \gamma \alpha\right\}\right) \leq C \gamma^{\frac{\theta}{m}} w\left(\left\{T_{*}(\vec{f})>\alpha\right\}\right)$.

We refer to [39] for the details and we just observe here that by standard methods, a consequence of this last theorem is the following result. 
Corollary 5. Fix exponents $1<p_{1}, \ldots, p_{m}, p<\infty$, such that $1 / p_{1}+$ $\cdots+1 / p_{m}=1 / p$, and $w \in A_{\infty}$. Then, for $\vec{f}=\left(f_{1}, \ldots, f_{m}\right)$ with each $f_{j}$ bounded and compactly supported

$$
\|T(\vec{f})\|_{L^{p}(w)} \leq C_{p, n} \prod_{j=1}^{m}\left\|M f_{j}\right\|_{L^{p_{j}}(w)} .
$$

Moreover, if $w \in A_{p_{0}}$, with $p_{0}=\min \left(p_{1}, \ldots, p_{m}\right)$, then

$$
\|T(\vec{f})\|_{L^{p}(w)} \leq C_{p, n} \prod_{j=1}^{m}\left\|f_{j}\right\|_{L^{p_{j}}(w)}
$$

and, in particular, $T$ extends as a bounded operator from $L^{p_{1}}(w) \times \cdots \times$ $L^{p_{m}}(w)$ into $L^{p}(w)$

\section{Concluding remarks and open problems}

We have systematically presented a study of general multilinear operators with kernels possessing singularities analogous to the ones of linear Calderón-Zygmund operators. In the process we have extended some known results and we have brought to surface some new ones involving, in particular, interpolation, endpoint estimates, and weighted norm inequalities.

There are many other aspects of the Calderón-Zygmund theory that one could pay attention to. We conclude by briefly mentioning some of them.

- Further $H^{p}$ results. Linear Calderón-Zygmund operators map $H^{1}$ into $L^{1}$. Similar endpoint results holds in the multilinear setting. For example $m$-linear Calderón-Zygmund operators maps $H^{1} \times$ $\cdots \times H^{1}$ into $L^{1 / m}$. See the work of Grafakos and Kalton [33].

- Multilinear T1 Theorem on other function spaces. A powerful way to prove the boundedness of linear Calderón-Zygmund operators on function spaces is by showing that they map appropriate smooth atoms into smooth molecules. See for example the works of Frazier, Torres, and Weiss [27], or Torres [58]. A similar approach is feasible in the multilinear setting. Some progress in this direction has been made by Bényi $[\mathbf{3}]$.

- Is there a multilinear Tb Theorem? Probably the conditions on the multilinear $T 1$ theorem can be further relaxed along the lines of the linear $T b$ theorem of David, Journé, and Semmes [19]. 
- Is there a multilinear theory for kernels $K$ that merely satisfy Hörmander's smoothness integrability condition? For some aspects of the theory this is not even known in the linear case.

- Is there a multiple weight theory? The most appropriate multilinear maximal function or multiple weights to work with in this direction are not yet clear.

- Multilinear singular integrals with rough kernels. Suppose that the kernel of a bilinear operator is given by

$$
K_{0}\left(y_{1}, y_{2}\right)=\Omega\left(\left(y_{1}, y_{2}\right) /\left|\left(y_{1}, y_{2}\right)\right|\right) /\left|\left(y_{1}, y_{2}\right)\right|^{2 n},
$$

where $\Omega$ is a function on $\mathbf{S}^{2 n-1}$ with mean value zero. Are $\Omega \in L^{q}$, $q>1$ or $\Omega \in L \log L$ sufficient to imply boundedness results?

As in the classical linear theory we may apply the method of rotations when $\Omega$ is an odd function to write

$$
\begin{aligned}
T_{\Omega}\left(f_{1}, f_{2}\right)(x) & =\iint \frac{\Omega\left(\frac{\left(y_{1}, y_{2}\right)}{\left|\left(y_{1}, y_{2}\right)\right|}\right)}{\left|\left(y_{1}, y_{2}\right)\right|^{2 n}} f_{1}\left(x-y_{1}\right) f_{2}\left(x-y_{2}\right) d y_{1} d y_{2} \\
& =\frac{1}{2} \int_{S^{2 n-1}} \Omega\left(\theta_{1}, \theta_{2}\right)\left\{\int_{-\infty}^{+\infty} f_{1}\left(x-t \theta_{1}\right) f_{2}\left(x-t \theta_{2}\right) \frac{d t}{t}\right\} d \vec{\theta} .
\end{aligned}
$$

The expression inside the curly brackets above is called the bilinear directional Hilbert transform (BHT) in the direction $\left(\theta_{1}, \theta_{2}\right)$. The $B H T s$ play the role of the linear directional Hilbert transforms. The uniform estimates on the bilinear Hilbert transforms were obtained by Thiele [56], Grafakos and $\mathrm{Li}[\mathbf{3 4}]$ and also by $\mathrm{Li}[\mathbf{4 5}]$ for a certain range of exponents when $n=1$. These results are based on ideas developed in [56]. Uniform estimates imply the boundedness of rough singular integrals with odd kernels. It would be interesting to know whether the corresponding higher dimensional bilinear Hilbert transforms are bounded. This is still an open question.

\section{References}

[1] D. Bekollé, A. Bonami, M. M. Peloso and F. Ricci, Boundedness of Bergman projections on tube domains over light cones, Math. Z. 237(1) (2001), 31-59.

[2] A. Benedek, A. P. Calderón and R. Panzone, Convolution operators on Banach space valued functions, Proc. Nat. Acad. Sci. U.S.A. 48 (1962), 356-365. 
[3] Á. BÉNYI, Bilinear singular integral operators, smooth atoms and molecules, submitted.

[4] G. Bourdaud, Une algèbre maximale d'opérateurs pseudo-différentiels, Comm. Partial Differential Equations 13(9) (1988), 1059-1083.

[5] A. P. Calderón and A. Zygmund, On the existence of certain singular integrals, Acta Math. 88 (1952), 85-139.

[6] M. Christ And J.-L. Journé, Polynomial growth estimates for multilinear singular integral operators, Acta Math. 159(1-2) (1987), 51-80.

[7] M. Christ and A. Kiselev, Absolutely continuous spectrum for one-dimensional Schrödinger operators with slowly decaying potentials: some optimal results, J. Amer. Math. Soc. 11(4) (1998), 771-797.

[8] R. R. Coifman, D. G. Deng and Y. Meyer, Domaine de la racine carrée de certains opérateurs différentiels accrétifs, Ann. Inst. Fourier (Grenoble) 33(2) (1983), 123-134.

[9] R. R. Colfman, S. Dobyinsky and Y. Meyer, Opérateurs bilinéaires et renormalisation, in: "Essays on Fourier analysis in honor of Elias M. Stein" (Princeton, NJ, 1991), Princeton Math. Ser. 42, Princeton Univ. Press, Princeton, NJ, 1995, pp. 146-161.

[10] R. R. Coifman and C. Fefferman, Weighted norm inequalities for maximal functions and singular integrals, Studia Math. 51 (1974), 241-250.

[11] R. R. Coifman and L. Grafakos, Hardy space estimates for multilinear operators. I, Rev. Mat. Iberoamericana 8(1) (1992), 45-67.

[12] R. R. Coifman, P.-L. Lions, Y. Meyer and S. Semmes, Compensated compactness and Hardy spaces, J. Math. Pures Appl. (9) 72(3) (1993), 247-286.

[13] R. R. COIFMAN AND Y. MeYer, On commutators of singular integrals and bilinear singular integrals, Trans. Amer. Math. Soc. 212 (1975), 315-331.

[14] R. R. Coifman and Y. Meyer, Commutateurs d'intégrales singulières et opérateurs multilinéaires, Ann. Inst. Fourier (Grenoble) 28(3) (1978), 177-202.

[15] R. R. Coifman and Y. Meyer, Au delà des opérateurs pseudodifférentiels, Astérisque 57, Société Mathématique de France, Paris (1978), 185 pp. 
[16] R. R. Coifman and Y. Meyer, Nonlinear harmonic analysis, operator theory and P.D.E., in: "Beijing lectures in harmonic analysis" (Beijing, 1984), Ann. of Math. Stud. 112, Princeton Univ. Press, Princeton, NJ, 1986, pp. 3-45.

[17] M. Cwikel and R. Kerman, Positive multilinear operators acting on weighted $L^{p}$ spaces, J. Funct. Anal. 106(1) (1992), 130-144.

[18] G. David AND J.-L. Journé, A boundedness criterion for generalized Calderón-Zygmund operators, Ann. of Math. (2) 120(2) (1984), 371-397.

[19] G. David, J.-L. Journé and S. Semmes, Opérateurs de Calderón-Zygmund, fonctions para-accrétives et interpolation, Rev. Mat. Iberoamericana 1(4) (1985), 1-56.

[20] S. Dobyinsky, Ondelettes, renormalisations du produit et applications a certains opérateurs bilineaires, Thése, Univ. Paris-Dauphine (1992).

[21] E. B. Fabes, D. S. Jerison and C. E. Kenig, Multilinear Littlewood-Paley estimates with applications to partial differential equations, Proc. Nat. Acad. Sci. U.S.A. 79(18) (1982), 5746-5750.

[22] C. Fefferman, Pointwise convergence of Fourier series, Ann. of Math. (2) 98 (1973), 551-571.

[23] C. Fefferman and E. M. Stein, Some maximal inequalities, Amer. J. Math. 93 (1971), 107-115.

[24] M. Frazier and B. Jawerth, Decomposition of Besov spaces, Indiana Univ. Math. J. 34(4) (1985), 777-799.

[25] M. Frazier And B. Jawerth, A discrete transform and decompositions of distribution spaces, J. Funct. Anal. 93(1) (1990), 34-170.

[26] M. Frazier, B. Jawerth and G. Weiss, "Littlewood-Paley theory and the study of function spaces", CBMS Regional Conference Series in Mathematics 79, published for the Conference Board of the Mathematical Sciences, Washington, DC, by the American Mathematical Society, Providence, RI, 1991.

[27] M. Frazier, R. H. Torres and G. Weiss, The boundedness of Calderón-Zygmund operators on the spaces $\dot{F}_{p}^{\alpha, q}$, Rev. Mat. Iberoamericana 4(1) (1988), 41-72.

[28] E. Gagliardo, On integral transformations with positive kernels, Proc. Amer. Math. Soc. 16 (1965), 429-434.

[29] J. E. Gilbert and A. R. Nahmod, Hardy spaces and a Walsh model for bilinear cone operators, Trans. Amer. Math. Soc. 351(8) (1999), 3267-3300.

[30] J. E. Gilbert and A. R. Nahmod, Bilinear operators with nonsmooth symbol. I, J. Fourier Anal. Appl. 7(5) (2001), 435-467. 
[31] J. E. Gilbert and A. R. Nahmod, Boundedness of bilinear operators with nonsmooth symbols, Math. Res. Lett. 7(5-6) (2000), $767-778$.

[32] L. Grafakos and N. J. Kalton, Some remarks on multilinear maps and interpolation, Math. Ann. 319(1) (2001), 151-180.

[33] L. Grafakos and N. J. Kalton, Multilinear Calderón-Zygmund operators on Hardy spaces, Collect. Math. 52(2) (2001), 169-179.

[34] L. Grafakos AND X. LI, Uniform bounds for the bilinear Hilbert transforms. I, submitted.

[35] L. Grafakos and R. H. Torres, Pseudodifferential operators with homogeneous symbols, Michigan Math. J. 46(2) (1999), $261-26$.

[36] L. Grafakos and R. H. Torres, Discrete decompositions for bilinear operators and almost diagonal conditions, Trans. Amer. Math. Soc. 354(3) (2002), 1153-1176.

[37] L. Grafakos and R. H. Torres, A multilinear Schur test and multiplier operators, J. Funct. Anal. 187(1) (2001), 1-24.

[38] L. Grafakos and R. H. Torres, Multilinear Calderón-Zygmund theory, Adv. in Math. 165 (2002), 124-164.

[39] L. Grafakos and R. H. Torres, Maximal operator and weighted norm inequalities for multilinear singular integrals, Indiana Univ. Math. J. 51 (2002) (to appear).

[40] R. Howard AND A. R. Schep, Norms of positive operators on $L^{p}$-spaces, Proc. Amer. Math. Soc. 109(1) (1990), 135-146.

[41] S. JANson, On interpolation of multilinear operators, in: "Function spaces and applications" (Lund, 1986), Lecture Notes in Math. 1302, Springer, Berlin, 1988, pp. 290-302.

[42] C. E. Kenig and E. M. Stein, Multilinear estimates and fractional integration, Math. Res. Lett. 6(1) (1999), 1-15.

[43] M. T. LaCey and C. M. Thiele, $L^{p}$ estimates on the bilinear Hilbert transform for $2<p<\infty$, Ann. of Math. (2) 146(3) (1997), 693-724.

[44] M. T. Lacey and C. M. Thiele, On Calderón's conjecture, Ann. of Math. (2) 149(2) (1999), 475-496.

[45] X. LI, Uniform bounds for the bilinear Hilbert transforms. II, submitted.

[46] Y. Meyer, "Ondelettes et opérateurs, I: Ondelettes", and "II: Opérateurs de Calderón-Zygmund", Actualités Mathématiques, Hermann, Paris, 1990. 
[47] Y. Meyer and R. R. Coifman, "Wavelets. Calderón-Zygmund and multilinear operators", Cambridge Studies in Advanced Mathematics 48, Cambridge University Press, Cambridge, 1997.

[48] C. Muscalu, T. TaO and C. M. Thiele, Multilinear operators given by singular multipliers, J. Amer. Math. Soc. 15 (2002), 469-496.

[49] F. Nazarov, S. Treil and A. Volberg, Cauchy integral and Calderón-Zygmund operators on nonhomogeneous spaces, Internat. Math. Res. Notices 1997(15) (1997), 703-726.

[50] J. Peetre, On convolution operators leaving $L^{p, \lambda}$ spaces invariant, Ann. Mat. Pura Appl. (4) 72 (1966), 295-304.

[51] J. Peetre, "New thoughts on Besov spaces", Duke University Mathematics Series 1, Mathematics Department, Duke University, Durham, N.C., 1976.

[52] S. Spanne, Sur l'interpolation entre les espaces $\mathcal{L}_{k}{ }^{p \Phi}$, Ann. Scuola Norm. Sup. Pisa (3) 20 (1966), 625-648.

[53] E. M. Stein, Singular integrals, harmonic functions, and differentiability properties of functions of several variables, in: "Singular integrals" (Proc. Sympos. Pure Math., Chicago, Ill., 1966), Amer. Math. Soc., Providence, R.I., 1967, pp. 316-335.

[54] E. M. SteIn, "Harmonic analysis: real-variable methods, orthogonality, and oscillatory integrals", Princeton Mathematical Series 43, Monographs in Harmonic Analysis III, Princeton University Press, Princeton, NJ, 1993.

[55] R. S. Strichartz, A multilinear version of the Marcinkiewicz interpolation theorem, Proc. Amer. Math. Soc. 21 (1969), 441-444.

[56] C. M. Thiele, A uniform estimate, submitted.

[57] R. H. TorRes, Continuity properties of pseudodifferential operators of type 1,1, Comm. Partial Differential Equations 15(9) (1990), 1313-1328.

[58] R. H. ToRres, Boundedness results for operators with singular kernels on distribution spaces, Mem. Amer. Math. Soc. 90(442) (1991), $172 \mathrm{pp}$.

[59] H. Triebel, "Theory of function spaces", Monographs in Mathematics 78, Birkhäuser Verlag, Basel, 1983.

[60] A. Youssfi, Bilinear operators and the Jacobian-determinant on Besov spaces, Indiana Univ. Math. J. 45(2) (1996), 381-396. 
Loukas Grafakos:

Department of Mathematics

University of Missouri

Columbia, MO 65211

U.S.A

E-mail address: loukas@math.missouri.edu

Rodolfo H. Torres:

Department of Mathematics

University of Kansas

Lawrence, KS 66045

U.S.A.

E-mail address: torres@math.ukans.edu 